(Aus der Universitäts-Augenklinik zu Heidelberg)

\title{
Weitere Beiträge zur Kenntnis seltener Missbildnngen.
}

Von

Prof. Eugen v. Hippel

in Heidelberg.

Mit Taf. I-III, Fig. 1-11.

I. Teratoma orbitae congenitum ${ }^{1}$ ).

(Hierzu Fig. 1-8 auf Taf. I u. II.)

Von Herrn Professor Marwedel in Aachen erhielt ich einen Orbitaltumor, den er im Zusammenhang mit dem Bulbus durch Exenteratio orbitae bei einem 5 Tage alten Kinde entfernt hatte. Die Geschwulst hatte hochgradigen Exophthalmus bedingt, die Lider bedeckten das Auge nicht, die Hornhaut war, wie ich allerdings nur aus der anatomischen Untersuchung schliesse, getrübt. Die Familienanamnese war belanglos, an dem Kinde selber wurden andere angeborene Fehler nicht wahrgenommen.

Das Präparat, das die Grösse eines mittlern Apfels besass (die Orbita war stark erweitert), wurde in Müllerscher Flüssigkeit fixiert und nach erfolgter Nachhärtung durch einen vertikalen Schnitt, welcher durch die temporale Hälfte des Bulbus ging, in zwei Teile zerlegt.

Hierbei ergab sich sofort, dass der Tumor nicht vom Bulbus ausging, sondern denselben nur einschloss. Auf dem Durchschnitt sah man massenhaft kleinere und grössere, zum Teil mit geronnener Flüssigkeit gefüllte cystische Räume sowie eine grosse Zahl eigentümlich durchscheinender Gewebsinseln, welche schon makroskopisch für hyalinen Knorpel gehalten wurden.

1) Über diesen Fall habe ich ganz kurz in der ophthalmologischen Sektion der Naturforscherversammlung zu Meran berichtet und die Mikrophotogramme gezeigt. Wie ich nachträglich erfahre, ist das Kind einige Wochen nach der Operation an "Dyspepsie" gestorben, eine Selktion konnte nicht gemacht werden.

r. Graefe's Archiv für Ophthalmologie, LXIIr. 1. 
Der Bulbus wurde im Zusammenhang mit dem Tumor in eine Schnittserie zerlegt, die Schnitte hatten die Grösse von ca. $4 / 3 \mathrm{~cm}$ (Dicke $30 \mu$ ), von den übrigbleibenden Teilen der Geschwulst wurden an verschiedenen Stellen kleinere Stücke entnommen und zu dünnen Schnitten $(10 \mu)$ verarbeitet; es ist demnach nicht sehr wahrscheinlich, dass irgendwelche wichtige Befunde übersehen worden sind.

Der Bulbus hat (an den Schnitten gemessen) einen antero-posterioren Durchmesser von 16, einen äquatorialen von $20 \mathrm{~mm}$, er ist also durch den Druck des Tumors von vorn nach hinten etwas abgeplattet, zeigt aber sonst normale Grössenverhältnisse. Der Sehnerv zieht, wie ans der Serie zu entnehmen ist, in vollkommen normaler Beschaffenheit durch den ganzen Tumor hindurch.

An der Cornea fehlt das Epithel vollständig. Bei Hämatoxylin-Eosinfärbung erseheint die Hornhaut bei schwacher Vergrösserung fast frei von getärbten Kernen - diffus rot. Bei starker Vergrösserung sieht man aber in den oberflächlichen Schichten schmale, blau gefärbte Striche zwischen den Lamellen; in den mittlern Lagen fehlt jede Färbung, und in den tiefsten, besonders nach dem Rande zu, trifft man normal aussehende Hornhautkörperchen an. Leukocyteninfiltration findet man ausschliesslich in der Limbusgegend; hier ist sie oben und unten sehr dicht und dringt in den hintern Hornhautschichten viel weiter vor als in den vordern; sie schneidet ziemlich geradlinig ab. In den vordersten Schichten ist sie an der Grenze des nicht mehr infiltrierten Gewebes besonders dicht, so dass man von der Andeutung eines Einwanderungsringes spreehen kann.

Der Befund ist wohl nur so aufzufassen, dass die Hornhaut, welche von den Lidern nicht bedeckt wurde, nahezu vollständig nekrotisch geworden ist, und dass die Leukocyteninfiltration bis an die Grenze der Nekrose heranreicht. Das Bild erinnert mich sehr an den von mir beschriebenen Fall von Keratitis neuroparalytica ${ }^{1}$ ) sowie an die Befunde, die Ollendorf²) bei länger fortgesetzter Luxation von Kaninchenaugen erhielt.

Das Endothel ist teils erhalten, teils in grossen Fetzen abgelöst; an diesen Stellen haben sich dann Leukocyten zwischen dieses und die Descemet gedrängt. Die vordere Kammer ist zum grössten Teil von mehrkernigen Leukocyten und Fibrinnetzen angefültt.

An der Vorderfläche der Iris, im Kammerwinkel und am Corpus ciliare findet reichliche Leukocytenauswanderung statt.

Im übrigen zeigen sämtliche Teile des Auges normale Verhältnisse

1) E. v. Hippel, Keratitis neuroparalytica. v. Graefe's Arch. f. Ophthalm. Bd. XLVII. S. 171.

2) Ollendorf, Über die Polle der Mikroorganismen bei der Entstehung der neuroparalytischen Keratitis. v. Graefe's Arch. f. Ophthalm. Bd. XLIX. S. 455 . 
bis auf eine kleine Anomalie im vordersten Abschnitt der Retina: hier findet man innerhalb der Nervenfaserschicht eine dichte Anhäufung von spindligen. Kernen, die der Hauptsache nach parallel zur Oberfläche der Netzhaut gestellt sind; ich kann den Befund nur für eine Wucherung von Gliazellen: halten, die aber durchaus keinen geschwulstartigen Charakter hat.

Der Tumor ist von einer derben Bindegewebskapsel - dem Periost der Orbita - eingeschlossen. An ihm selbst fällt schon bei schwacher Vergrösserung und oberflächlicher Betrachtung die enorme Vielgestaltigkeit der ihn zusammensetzenden Elemente auf. Dieselben sind regellos durcheinandergeworfen und in eine mehr oder weniger kernreiche bindegewebige Grundsubstanz eingelagert. Dieselbe hat vielfach den Charakter des embryonalen Bindegewebes. Folgende Bestandteile lassen sich unterseheiden:

1. Zentralnervensystem: dasselbe ist in den verschiedenen Gegenden der Geschwulst sehr angleich reichlich vorhanden, in den Schnitten, welche durch die Mitte des Bulbus gehen, trifft man es an vielen Stellen an. Fig. 3 zeigt einen $10 \mathrm{~mm}$ langen und ungefähr $2 \mathrm{~mm}$ breiten Streifen vom Charakter des Gliagewebes; derselbe ist an der Oberfläche von einer mehr= schichtigen Zelllage bedeckt und grenzt an einen grossen Hohlraum. Unmittelbar unter derselben sieht man helle, mit Eosin schwachrötlich gefärbte Partien, die bei starker Vergrösserung wie quer getroffene, sehr feine Nervenfasern aussehen; dann folgt ein Band dicht gedrängter, scharf konturierter runder Zellen vom Charakter der Gliazellen, und auf diese wieder ein sehr feiner Faserfilz. Zweifellose Ganglienzellen konnten ebensowenig nachgewiesen werden wie markhaltige Nervenfasern.

In unmittelbarer Nähe dieses Bezirks, aber auch an zahlreichen andern Stellen sieht man, wie Fig. 4 zeigt, zottige Bildungen, die man wohl mit Recht als Plexus choroidei deuten darf.

Sehr reichlich und an den verschiedensten Stellen erscheinen danr innerhalb der Geschwulst Bezirke (Fig. 5), wo in einer gliösen Grundsubstanz Inseln typischen Neuroepithels auftreten. Dieselben stellen sich im Schnitt in verschiedenster Form, als Bänder, Halbmonde, sowie in typischer Ring- bzw. Rosettenform dar. An einzelnen Stellen sieht man, wie diese Neuroepithelstreifen sich allmählich verdünnen and in eine einschichtige Lage kubischer Zellen ïbergehen. Letztere ist mehrfach intensiv pigmentiert und entspricht in ihrem Aussehen völlig dem normalen retinalen Pigmentepithel. Derartige Streifen and Inseln von Pigmentepithel kommen an ganz verschiedenen, weit voneinander entfernten Stellen des Tumors vor.

2. Eine rudimentäre Angenanlage (Fig. 1a, Fig. 2) trifft man etwas nach hinten und oben von dem normalen Bulbus innerhalb des $\mathrm{Tu}$ mors an; sie sieht im Durchschnitt nicht regelmässig rund, sondern etwas eckig aus, misst $3{ }_{4}^{1 /}: 2^{1} / 4 \mathrm{~mm}$ und ist besonders bemerkenswert dadureh, dass die Retina in inverser Anordnung der Schichten aussen, das Pigmentepithel dagegen innen liegt; einwärts vom Pigmentepithel kann man eine unvollständige Chorioidea von embryonalem Zelltypus erkennen; im übrigen sieht man im Schnitt im Lumen der Augenblase ganz verschiedenartige Gewebe; die Serie zeigt ausserdem, dass es sich bei dieser Augenblase um kein auch nur einigermassen kugliges, sondern ein ganz unregelmässig kom- 
primiertes Gebilde handelt. Von einer Linsenanlage ist nichts zu erkennen. Die eigentümliche Lagerung der einzelnen Membranen kann man sich in folgender Weise zu stande gekommen denken: eine ursprünglich normal gestaltete sekundäre Augenblase, die vorn aber weder durch Linse noch durch Hornhaut abgeschlossen ist, kann dureh ein gegen ihre hintere Wand andrängendes Tumorgewebe handschuhfingerartig umgestülpt werden, so dass die äussern Membranen nach innen gelangen und umgekehrt. Im ganzen hat die Augenanlage einen ziemlich hohen Grad von Differenzierung erlangt, wenn auch die Retina noch durchaus embryonalen Typus zeigt (s. Fig. 2).

3. Epidermis und ihre Abkömmlinge (Fig. 6): man trifft eine ziemlich grosse Zahl von Hohlräumen, die mit einer deutlichen Epidermis ausgekleidet sind. Den Inhalt der Hoblräume bilden abgestossene, zum Teil verhornte und zu Klumpen zusammengebackene Epidermiszellen; ausserdem trifft man an der Oberfläehe eines grösseren Hohlraums einen Streifen Haut mit Epidermis und den Anlagen von Haaren und Talgdrüsen.

4. Hyaliner Knorpel: durch den ganzen Tumor zerstreut findet man kleinere und grössere Stücke desselben; zum Teil sind sie ausserordentlich zellenreich - mehr embryonaler Typus -, zum Teil haben sie mehr das Aussehen fertigen, hyalinen Knorpels.

5. Knochen: er ist nur. sehr spärlich vertreten, die Knochenbälkchen sind klein, Markräume und Osteoblasten deutlich.

6. Muskelgewebe: glatte Muskelfasern sieht man an verschiedenen Stellen, die erhaltenen Augenmuskeln sind in der Peripherie des Tumors nachweisbar.

7. Nekrotische Partien, zum Teil mit reichlicher Kalkablagerung finden sich an den verschiedensten Stellen der Geschwulst.

8. Grössere Blutungen trifft man besonders reichlich in den hintersten Partien des Tumors.

9. Stark erweiterte Blutgefässe sind ein häufiger Befund; dieselben bilden manchmal vollständige Konvolute, die Getässräume sind durch schmale Bindegewebsbrïcken voneinander geschieden. Diese Stellen haben weitgehende Ähnliehkeit mit einem kavernösen Angiom.

10. Hohes Cylinderepithel mit basal gelagertem Kern und einem breiten lichten Saum, der dem Lumen zugekehrt ist, kleidet eine grössere Zahl von cystischen Hohlräumen aus (Fig. 7). Ein Teil der Zellen gilbt mit Hämatoxylin ausgesprochene Schleimreaktion (Fig. 7). Hier und da trifft man Durchschnitte von Hohlräumen, wo der Kern soleher hoher $\mathrm{Cy}$ linderzellen nach dem Lumen, der lichte Saum nach der Wand zu gerichtet ist. Hier muss also eine ähnliche Einstülpung wie bei der rudimentären Augenanlage stattgefunden haben. Sehr bemerkenswert ist es, dass das hohe Cylinderepithel vielfach ganz unvernittelt in niedriges kubisches übergeht, so dass derselbe Hohlraum von zwei verschiedenen Epithelarten ausgekleidet ist. Man kann die Cylinderepithelien wohl nur als Bestandteile des Respirations- bzw. Verdauungstractus ansprechen, Fig. 7 erinnerte lebhaft an das Aussehen von Darmzotten.

11. Unzweifelhaftes Drüsengewebe sieht man an den verschiedensten Stellen (Fig. 8); es handelt sich um acinöse Formen, die man wohl nicht einer bestimmten Drüsenform des normalen Körpers ohne weiteres gleich- 
stellen kann. Eine Ähnlichkeit mit fötalem Schilddrüsengewebe ist allerdings vorhanden.

12. Follikel: vereinzelt kommen Anhäufungen von dicht gedrängten, dunkel getärbten einkernigen Zellen vor, die eine unverkennbare Ähnlichkeit mit Lymphfollikeln besitzen.

13. Ausser den beschriebenen Elementen finden sich innerhalb der Geschwulst noch zahlreiche epitheliale Bildungen, die sich mit keinem normalen Gewebe direkt identifizieren lassen; dieselben haben stellenweise in ihrer Anordnung gewisse Ähnlichkeit mit Carcinomgewebe. In allen Geschwulstelementen lassen sich - - sogar vielfach recht zahlreich - Mitosen erkennen, was für ein rasches Wachstum des Tumors spricht.

\section{Epikrise.}

Bei einem 5 Tage alten Kinde findet sich eine apfelgrosse Geschwulst der Orbita; dieselbe ist also während des fötalen Lebens entstanden. Wie lange vor der Geburt sie angefangen hat zu wuchern lässt sich nicht angeben; andere Erfahrungen (siehe unten) lehren, dass derartige Tumoren enorm rasch wachsen können.

Der Tumor ist aus Geweben zusammengesetzt, welche Bestandteile aller drei Keimblätter enthalten: Zentralnervensystem, Augenanlagen, Epidermis, Haarbälge, embryonales Bindegewebe, hyaliner Knorpel, Knochen, glatte Muskulatur, Blutgefässe, Follikel, Elemente des Respirations- bzw. Verdauungstractus, Drüsengewebe. Diese sämtlichen Elemente liegen regellos durcheinander; sie haben grossenteils ausgesprochen embryonalen Gewebscharakter. $\mathrm{Zu}$ der vollständigen Ausbildung auch nur eines Organs ist es nicht gekommen.

Die histologische Diagnose kann nur lauten: Angeborenes Teratom der Orbita. In der Literatur fand ich, soweit ich mich bis zu meinem Meraner Vortrag hatte orientieren können, nur einen analogen Fall, der vor 29 Jahren von Weigert und Broër ${ }^{1}$ ) mitgeteilt wurde.

Bei einem einen Tag alten Kinde fand sich ein apfelsinengrosser Tumor der rechten Orbita; auf der Höhe des Tumors der Bulbus, Cornea leicht getrübt, nach fünf Tagen Hypopyon, Lidödem. Punktion der Geschwulst, aus welcher sich eine grosse Menge klarer gelblicher Flüssigkeit entleert; der Tumor collabiert zur Hälfte und wird exstirpiert. Tod nach zwei Tagen an fibrinös-eitriger Periearditis.

Von der Innenfläche der eröffneten Cyste lässt sich geschichtetes Flimmerepithel abschaben, man findet eine Anzahl kleinerer und

1) Broër. und Weigert, Teratoma orbitae congenitum. Virchows Arch. Bd. LXVII. S. 518. 
grösserer Cysten; nach hinten zu Fettgewebe, innerhalb desselben ein wurstförmiges Gebilde vom Aussehen einer Darmschlinge; ferner Knorpel und Knochenteile, von denen einer bei einiger Phantasie an einen Extremitätenknochen erinnert. Mikroskopisch: die Hauptmasse Fettgewebe, ausserdem Bindegewebe, hyaliner Knorpel, zum Teil in Verknöcherung; epitheliale Massen, am seltensten solche rom Charakter der Epidermis, ferner Auskleidung mit einfachem Cylinderepithel, schleimige Massen an der Oberfläche. In dem darmähnlichen Gebilde Schläuche vom Aussehen der Lieberkühn'schen Drüsen.

Also auch in diesem Falle Elemente aller drei Keimblätter. Von Zentralnervensystem und Augenanlage wird nichts berichtet, doch ist $\mathrm{zu}$ berücksichtigen, dass derartige Dinge übersehen werden können, wenn man nicht Serienschnitte macht oder wenigstens sehr zahlreiche Teile der Geschwulst untersucht; es ist also nicht ausgeschlossen, dass sie auch in jenem Falle tatsächlich vorhanden waren.

Nachträglich habe ich nun noch folgende Fälle ermitteln können, welche wohl hier einzureihen sind.

Lawson ${ }^{3}$ ) fand bei einem neugeborenen Knaben das rechte Auge durch einen orbitalen Tumor stark vorgetrieben. Das Kind starb drei Monate später unter Krämpfen. Bei der Sektion zeigte sich ein Tumor, weleher vom Keilbeinkörper ans sich gegen die Orbita und das Gehirn vorgeschoben hatte. Die Geschwulst enthielt zahlreiche Cysten verschiedener Grösse, welche von Plattenepithel ausgekleidet und mit kolloidem Inhalt gefüllt waren, mehrere Inseln von hyaliner Knorpelsubstanz, mitunter von mehrfacher Lage von Spindelzellen eingeschlossen. Die Hauptmasse bildete zellreiches embryonales Bindegewebe.

Es handelt sich also auch hier zweifellos um eine Mischgeschwulst, in welcher allerdings nur die Elemente zweier Keimblätter nachgewiesen sind. Ob die Geschwulst wirklich vom Keilbeinkörper ausgegangen ist und diesen nicht vielmehr sekundär ergriffen hat, vermag ich nicht $\mathrm{zu}$ entscheiden.

Courant ${ }^{2}$ sah bei einem sonst normalen Neugeborenen eine Vortreibung des linken Auges um $2 \mathrm{~cm}$. Dasselbe ist eingeschlossen von einem Tumor, welcher die ganze Orbita auszufüllen scheint und die Lider voneinander drängt. Die Hornhaut war getrübt und geschwürig, es bestand Hypopyon. Der Tumor wurde in toto ausgeschält, was ziemlich leicht gelang, das Kind überstand den Eingriff gut und entwickelte sich rortrefflich weiter.

Der N. opticus verlief frei durch die Geschwulst, innerhalb der letztern

1) Lawson, Congenital tumour of the orbit, complete exophthalmos in a child two days old, removal the of eye. Lancet 1885 . Vol. II. p. 684.

2) Courant, Über eine seltene Orbitalgeschwulst des Neugeborenen. Centralblatt f. Gynäkolog. 1893. S. 740. 
fand sich eine grosse buckelförmige Cyste mit serösem, ferner eine kleinere mit schleimigem Inhalt, ausserdem knorplige und knochenharte Teile, in der Mitte ein nussgrosser Knochenkern.

Mikroskopisch besteht die Wand der grossen Cyste aus jungem Bindegewebe mit Inseln von Fett, die Innenfläche ist von Cylinderepithel ausgekleidet, welches zahlreiche Ausstülpungen in die Umgebung macht. In der andern Cyste ist mehrschichtiges niedriges Epithel vorhanden, in der Wand Drüsenacini, Talgdrüsen und Haarbalgdurchschnitte, endlich trifft man hyalinen Knorpel und daraus hervorgehenden Knochen.

Von Elementen des Nervensystems oder rom Pigmentepithel wird nichts berichtet, es ist aber auch keine systematische Untersuchung der ganzen Geschwulst vorgenommen worden.

Die von dem Autor festgestellten Gewebe genïgen aber bereits völlig, um Abkömmlinge aller drei Keimblätter mit Sicherheit zu diagnostizieren. Der Fall ist also unzweifelhaft den Teratomen zuzurechnen. Der Satz des Verfassers „die Beschaffenheit der Geschwulst weist darauf hin, dass der embryonale Ursprung der Geschwulst in einer Anomalie bei der Bildung der sekundären Augenblase zu suchen ist", erscheint mir etwas unklar, ich kann den Standpunkt des Autors daraus nicht erkennen.

Lagrange ${ }^{1}$ ) beschreibt wegen seiner extremen Seltenheit folgenden Fall: 20 Tage altes Kind mit einem grossen Tumor der rechten Orbita, welcher das Ange nach unten innen verdrängt hat. (Die Abbildung entspricht genau den von Weigert-Broër und von Courant gegebenen.) Die Cornea trübte sich schon am Tage nach der Geburt, unter dem obern Lid entleerte sich einmal sanguinolente Flüssigkeit, eine gleiche wurde auch durch Punktion gewonnen. Die Geschwulst war nach ärztlichem Bericht während der ersten 20 Tage rapid gewachsen, ihre Oberfläche war ulceriert, ebenso die Cornea.

Sonstige Anomalien waren bei dem Kinde nicht vorhanden. Die Geschwulst wurde mit dem Thermokauter in toto exstirpiert; der Stiel, mit welchem sie hinten endigte, bestand noch aus Tumorgewebe. Tod drei Tage nach der Operation. Sektion: Hämatom über dem rechten Hinterhauptsund Schläfenlappen (Folge einer schweren Geburt), im übrigen Gehirn normal. Durch die Fissura orbitalis superior ragt ein nussgrosses Stüek des Tumors gegen die Schädelbasis.

Die histologische Untersuchung ergab: Bulbus bis auf die HypopyonKeratitis normal, Sehnerv normal. 'Der grösste Teil der Geschwulst besteht aus embryonalem Nervengewebe, die Zellen entsprechen den frühen Entwicklungsstadien von Gehirn und Rückenmark mit zartem faserigem Zwischengewebe, diese Zellen sind umgeben von Zellen, die den Typus des

1) Lagrange, Tumeur congénitale embryonnaire à tissus multiples (nerveux et conjonctif) de l'orbite. Arch. d'Ophtalm. Tom. XV. p. 563. 1895. 
embryonalen Bindegewebes zeigen; hier sind also Bestandteile von zwei Keimblättern nachgewiesen.

Über andere. Gewebselemente wird nicht berichtet, nur ist bei der makroskopischen Schilderung von einigen „falschen" Oysten die Rede, welche durch Erweichung von Gewebselementen entstanden seien. Ob die Untersuchung so vollständig ist, dass andere Gewebselemente nicht unentdeckt bleiben konnten, wage ich nicht zu entscheiden. Ich möchte aber auch so den Tumor den Teratomen zurechnen. Da zweifelloses Nervengewebe von embryonalem Charakter nachgewiesen ist, kann es sich nur um eine völlig abgeschnürte Encephalocele oder ein Teratom handeln. Die normale Beschaffenheit des Gehirns sowie das äusserst rasche Wachstum, die exquisite Malignität des Tumors sprechen mit Entschiederheit für das letztere.

Wahrscheinlich gehört hierher auch ein leider nur klinisch beobachteter Fall von Polignani ${ }^{1}$, den er als cystisches Fibrosarkom bezeichnet.

Ein neugeborener Knabe hatte auf der linken Seite einen Orbitaltumor, welcher in einigen Wochen zur Zerstörung des Augapfels führte und die Grösse einer kleinen Mandarine erreichte, die Diagnose cystisches Sarkom wurde auf Grund einer Probepunktion gestellt. Von einem operativen Eingriff wurde abgesehen, das Kind starb nach 6 Monaten, Sektion konnte nicht gemacht werden.

Weiter hat Ewetzky") einen Fall unter der Bezeichnung "Cephaloma orbitae" beschrieben, der mir ebenso wie der vorige nur im Referat (Natanson) zugänglich ist.

Bei einem Kinde von $4^{1} / 2$ Monaten war die rechte Orbita von einem grossen Tumor angefüllt, an dessen Spitze ein Bulbusrudiment festgestellt wurde. Es wurde die Exenteratio orbitae gemacht, dabei blieben aber Tumormassen zurück, die rasch wieder zu wachsen anfingen. „Die im Durchschnitt gelbliche Geschwulst von schwammiger Konsistenz misst 4:5,5 cm; enthält stellenweise knochenharte Plaques, etwas schwarzes Pigment und besteht vorwiegend aus Binde- und Nervengewebe; im fibrösen Bindegewebe findet sich auch Fett, Knorpel, Knochen, Kalk. In der Neuroglia sind unechte Kerne und grosse pyramidale, runde, spindelförmige, keulenförmige und andere Zellen enthalten." Verschiedenartige Cysten, zum Teil mit Flimmerepithel, wurden gefunden.

„Es handelt sich also um einen Hirnbruch, der nicht mehr im Zusammenhang mit dem Gehirn steht und zu den Teratomen zu zählen ist."

1) Polignani, Fibrosarcoma cistico dell'orbite di genese fetale con diffusione all'occhio. Napoli. Tip. Tocco. 1901. Ref. in Rev. gén. d'Ophtalm. 1902. p. 521.

2) Ewetzky, Cephaloma orbitae. Moskauer augenärztliche Gesellschaft. Wratsch. XXI. p. 690. Ref. in Michels Jahresbericht 1904 von Natanson. 
Dass hier ein echtes Teratom vorliegt, erscheint bei seiner $\mathrm{Zu}$ sammensetzung aus charakteristischen Elementen aller drei Keimblätter zweifellos, weniger sicher dürfte die Annahme eines Hirnbruchs sein, doch wäre es nur möglich, über diesen Punkt etwàs bestimmtes auszusagen, wenn man die Mitteilung im Original zur Verfügung hätte.

Ahlfeld ${ }_{1}^{1}$ ) hat noch einen Fall beobachtet, der mit dem meinigen und den vorstehenden einige Ähnlichkeit hat; v. Duyse hat denselben in seinem Buche abgebildet. Dort handelt es sich um Implantation eines rudimentären Fötus in die Orbita, bei welchem man eine Extremität sehr deutlich erkennt. Ausserdem bestand in jenem Falle eine grosse Encephalocele über der Nasenwurzel.

Wir haben also die Fälle von Broër-Weigert, Courant, Ewetzky, Ahlfeld und den meinigen wegen der Anwesenheit von Elementen aller drei Keimblätter als sichere Teratome zu diaguostizieren. Die klinisch übereinstimmenden von Lawson und Lagrange sind Mischgeschwülste mit Elementen zweier Keimblätter; wir werden im weitern sehen, dass diese von den Teratomen nicht scharf zu trennen sind.

Dagegen ist aus dieser Gruppe ein Fall zu streichen, den Gallenga ${ }^{2}$ ) in einer neuern Arbeit unter die Teratome, rechnet. Der Irrtum ist nur durch sprachliche Schwierigkeiten zu erklären. Barranechea ${ }^{3}$ ) beschreibt einen Orbitaltumor, ein Fibrosarkom; in der Arbeit steht der Satz: „übrigens findet man Muskelgewebe, Gefässe, Nerven- und Drüsengewebe, alles Elemente, welche der Tumor in seinem Wachstum zusammengebracht hat." Gallenga hat daraus offenbar geschlossen, dass diese Elemente Bestandteile des Tumors seien, während es sich um die normalen Gebilde handelt, die vom Tumor umwachsen sind.

Die bisher bekannt gewordenen Teratome der Orbita haben folgende klinische Eigentümlichkeiten:

Regelmässig waren sie bei der Geburt schon als grosse Tumoren vorhanden, die zu einer so starken Protrusio bulbi geführt hatten, dass die Cornea nicht mehr von den Lidern bedeckt war und sich deshalb rasch trübte und eitrig zerfiel. Im übrigen wurde der Bulbus

1) Ahlfeld, Die Missbildungen des Menschen. Atlas: Taf. VI. Fig. 11. 1880.

2) Gallenga, Contribuzione allo studio dei tumori congeniti dell' orbita (fibrolipoma congenito). Arch. di Ottalm. VI. 3.

3) Barranechea, Beiträge zur Geschwulstlehre des Auges. Centralbl. f. Augenheilk. 1889. S. 101. 
sowie der Sehnerv regelmässig normal befunden, beide nahmen an der Geschwulstbildung keinen Anteil.

Die Tumoren waren durch ein äusserst rapides Wachstum, also einen hohen Grad von Malignität ausgezeichnet. Da sie fast ausschliesslich aus Geweben von embryonalem Charakter mit zahlreichen Mitosen zusammengesetzt sind, so kann dies Verhalten nicht wundernehmen.

Fast in allen Fällen gingen die Kinder in den ersten Lebenswochen zu Grunde, nur der Fall von Courant zeigt, dass eine rechtzeitige Operation unter günstigen Verhältnissen Heilung herbeiführen kann.

Hereditäre Belastung fehlte in allen Fällen, auch bestanden bei den Kindern keine andern angeborenen Anomalien, mit Ausnahme des auch sonst abweichenden Falles von Ahlfeld, wo eine grosse Encephalocele über der Nasenwurzel vorhanden war.

Was die histologische Zusammensetzung der Geschwülste angeht, so handelt es sich um ausgesprochene Mischgeschwülste: fünfmal sind Elemente aller drei Keimblätter nachgewiesen, zweimal die von zweien (Ektoderm und Mesoderm). Nicht in allen Fällen ist die Untersuchung eine so vollständige, dass die Anwesenheit noch anderer Elemente, als die von den Autoren geschildert wurden, auszuschliessen wäre. Die grösste Mannigfaltigkeit der Befunde zeigt wohl mein Fall. Ganz besonders zu betonen ist, dass in allen Fällen ein grosser Teil der vorhandenen Gewebselemente ausgesprochen embryonalen Charakter zeigt. Zur Ausbildung vollständiger Organe ist es in keinem Falle gekommen: bei Broër und Weigert wurde ein darmähnliches Gewebsstück, in meinem Falle eine rudimentäre Augenanlage nachgewiesen.

Wenn man sich mit der Genese dieser Tumoren und ihrer Bedeutung für die Lehre von den Missbildungen befassen will, so ist es notwendig, wenigstens in aller Kürze auf einige neuere Arbeiten einzugehen, die sich mit ähnlichen Geschwülsten an andern Körperstellen beschäftigen. Hier kommen vor allen Dingen die Abhandlungen von Schwalbe ${ }^{1}$ ) über den Epignathus und von Askanazy ${ }^{2}$ ) über die Dermoidcysten des Eierstocks in Betracht, ferner das Referat,

1) Schwalbe, Der Epignathus und seine Genese. Zieglers Beiträge. Bd. XXXVI. S. 242.

$\left.{ }^{2}\right)$ Askanazy, Die Dermoidcysten des Eierstocks, ihre Geschichte, ihr Bau und ihre Entstehung, sowie ihre Beziehung zu verwandten pathologischen Bildungen. Biblioth. Med. C. Heft 19. 1905. 
welches Fischel ${ }^{1}$ ) der Deutschen Pathologischen Gesellschaft in Karlsbad erstattet hat.

Schw al be unterscheidet beim Epignathus folgende vier Gruppen:

1. An dem Gaumen oder in der Nachbarschaft ist der Nabelstrang eines zweiten Fötus befestigt; dieser kann mehr oder weniger gut ausgebildet sein.

2. Aus der Mundhöhle hängen Körperteile eines zweiten Fötus, die sich ohne weiteres als ausgebildete Organe erkennen lassen.

3. Aus der Mundhöhle ragt eine formlose Masse, anatomisch gekennzeichnet als Teratom (Produkte aller drei Keimblätter in regelloser Anordnung).

4. Ein grösserer oder kleinerer Tumor befindet sich am Gaumen oder in der Mundhöhle, die Untersuchung ergibt die Zusammensetzung vom Typus der Mischgeschwulst.

Zur Erklärung dieser verschiedenen Formen hat man in den meisten ältern Arbeiten angenommen, dass die Gruppen 1 und 2 als Foetus in Foetu, also als Doppelbildungen anzusehen seien, während es sich in den Gruppen 3 und 4 um die Folgen von Verlagerungen solcher Gewebselemente handelt, die dem Körper des Autositen entstammen.

Dem gegenüber sucht Schwalbe auf Grund der Ergebnisse besonders entwicklungsmechanischer Forschungen eine einheitliche Erklärung aller vier Gruppen zu gewinnen, in welcher der Gegensatz "autochthone" oder "heterochthone" Bildung (Arnold ${ }^{2}$ ) bedeutungslos wird. Dies ist möglich, wenn man der Marchand-Bonnetschen Blastomerentheorie eine etwas erweiterte Fassung gibt. Dieselbe nimmt zur Erklärung der Teratome an, dass eine aus dern Zusammenhang ausgeschaltete Blastomere an irgend eine Stelle des weiter wachsenden embryonalen Organismus zu liegen kommt und hier entweder als ruhender Keim verbleibt, um sich erst nach langer Latenz weiter zu entwickeln, oder bereits im fruihen embryonalen Stadium annähernd gleichzeitig mit dem normalen Organismus eine selbständige Entwicklung beginnt.

Geht man von dem Stadium der befruchteten Eizelle aus, die ihre erste Teilung in zwei Furchungskugeln vollzogen hat, so sind diese

1) Fischel, Über den gegenwärtigen Stand der experimentellen Teratologie. Referat erstattet der pathol. Gesellschaft Karlsbad 1902.

2) Arnold, Über behaarte Polypen der Rachenmundhöhle und deren Stellung zu den Teratomen. Virchows Arch. Bd. CXI. S. 176. 
beiden, wie die experimentelle Entwicklungsgeschichte lehrt, totipotent, d.h. jede derselben besitzt die Fähigkeit, einen ganzen normalen Organismus zu liefern. Diese Totipotenz der Blastomeren nimmt mit fortschreitender Teilung derselben ab, und es wird infolgedessen auf einem gewissen Stadium eine Blastomere nicht mehr die Fähigkeit besitzen, einen normalen Organismus auszubilden, wohl aber noch einzelne Teile eines solchen. Gehen wir weiter, so wird eine ausgeschaltete Zelle oder eine Gruppe von solchen vielleicht noch die Fähigkeit haben, durch Selbstdifferenzierung Gewebselemente zu produzieren, welche histologisch verschiedenen Keimblättern entsprechen, aber nicht mehr vollständige Organe bilden und schliesslich, wenn die Sonderung in Keimblätter schon einen höhern Grad der Vollständigkeit erreicht hat, kann durch Ausschaltung und Inklusion einzelner Zellen später eine Mischgeschwulst entstehen, die eventuell nur die Elemente von zwei Keimblättern enthält.

Schwalbe nennt den spätesten Zeitpunkt, an den man die Entstehung der Missbildung verlegen kann, die "teratogenetische Terminationsperiode" und kommt für den Epignathus zu dem Schluss: ,je komplizierter der Bau des Epignathus, desto früher ist im allgemeinen die teratogenetische Terminationsperiode zu setzen".

Die Möglichkeit, die vier verschiedenen Typen des. Epignathus einheitlich zu erklären, liegt also darin, dass man sie sämtlich auf die Ausschaltung embryonalen Keimmaterials zurückführt. Nur der Zeitpunkt, an dem dieselbe stattfindet, und die hierdurch bedingte ungleiche Potenz des ausgeschalteten Materials entscheidet darüber, ob schliesslich ein fast vollständig ausgebildeter zweiter Organismus, Teile eines solchen, ein Teratom oder eine einfache Mischgeschwulst entstehen wird.

Wir können für die angeborenen Orbitaltumoren eine ähnliche, wenn auch nicht so vollständige Reihe aufstellen wie für den Epignathus. Ausscheiden werden wir bei solcher Betrachtung diejenigen angeborenen Tumoren, welche ihrer Zusammensetzung nach aus den normalen Orbitalgebilden hervorgegangen sein können, also Fibrome, Sarkome, Lipome, Chondrome, dagegen dürfen wir noch solche einbeziehen, die ihrer histologischen Beschaffenheit nach nur aus Keimen abgeleitet werden können, die durch pathologische Entwicklungsvorgänge in die Orbita verlagert sind, wenn dieselben auch klinisch von den oben zusammengestellten Fällen in gewissen Punkten abweichen. 
Dahin dürfte wohl ein von Vanzetti ${ }^{1}$ ) veröffentlichter Fall zu rechnen sein.

Bei einem dreijährigen Kinde wurde ein Orbitaltumor nach Enucleation des sehtïchtigen Auges (!) entfernt. Derselbe bestand nur aus den charakteristischen Gliazellen mit spärlichem Protoplasma, grossem Kern und den zahlreichen strahligen, netzartig sich kreuzenden Ausläufern. Die vorhandenen Gefässe sind der Hauptsache nach nur Endothelröhren. Der Tumor wird als primäres Gliom der Orbita aufgefasst und für ein Unicum gehalten.

Setzen wir diesen der Hauptsache nach nur aus den Elementen eines Keimblatts bestehenden Tumor ans untere Ende der Reihe, so würden auf ihn die Mischgeschwülste mit den charakteristischen Elementen zweier Keimblätter folgen. An diese schliessen sich solche an, in denen man deutliche Produkte aller drei Keimblätter nachweisen kann. Bei dieser letzten Gruppe könnte man noch zwei Unterabteilungen bilden, je nachdem rudimentäre Organe gebildet sind oder nicht. Als oberste Stufe der Reihe würde der Fall Ahlfelds zu betrachten sein, wo die Geschwulst deutliche Organteile erkennen lässt. Ein mit einer Nabelschnur in der Orbita eingepflanzter Fötus, welcher die Analogie mit dem Epignathus fertig durchführen liesse, ist bisher meines Wissens nicht beobachtet worden.

Welche Zeit der Entwicklung wir als die ,teratogenetische Terminationsperiode" unserer orbitalen Teratome anzusetzen haben, vermag ich nicht näher anzugeben, ich kann mir aber eine Verlagerung von Keimen in diese Gegend nur in den frühesten Entwicklungsstadien vorstellen.

Es ist zu beachten, dass die Gewebe unserer Teratome der Hauptsache nach durchaus embryonalen Charakter haben, es ist also anzunehmen, dass der Keim, aus dem sie entstanden sind, erst nach längerer Latenz begonnen hat sich zu entwickeln; es ist gar nicht ausgeschlossen, dass das Wachstum erst einige Wochen vor der Geburt begonnen hat, man kann das wenigstens für möglich halten, wenn man die ausserordentlich rasche Grössenzunahme der Geschwülste post partum berücksichtigt. Jedenfalls stimmen diese Teratome der Orbita mit den Teratomen der Mundhöhle (Epignathus, 3. Gruppe) darin überein, dass sie bereits bei der Geburt vorhanden sind, und unterscheiden sich dadurch in höchst auffallender Weise von den Teratomen der Ovarien. In den weiblichen Keimdrüsen sind die

1) Vanzetti, Glioma primitivo della cavità orbitaria. Ann. di Ottalm. e Lavori della clin. ocul. di Napoli. XXX. p. 33. Referat in Michels Jahresb. 1901 von Berlin-Palermo. 
Teratome relativ am häufigsten beobachtet worden und hier ist nach den Angaben von Askanazy ${ }^{1}$ noch niemals beim Fötus oder Neugeborenen ein solides Teratom zur Beobachtung gekommen, sondern diese Tumoren haben sich ausnahmslos im spätern Leben, hauptsächlich in den Jahren der Geschlechtsreife des Weibes entwickelt. Übereinstimmend mit den orbitalen Teratomen ist dagegen ihre histologische Zusammensetzung, auch bei ihnen finden sich wesentlich embryonale Gewebsformen, ferner hier wie dort das rasche, nahezu unbegrenzte Wachstum dieser Tumoren.

Die Teratome und die ihnen nahestehenden Dermoidcysten des Eierstocks haben seit langem das Interesse der Forscher erregt. Askanazy bringt in der Einleitung eine sehr eingehende historische Übersicht über die Auffassungen, die zu verschiedenen Zeiten massgebend waren.

Hier sei daraus nur folgendes wiedergegeben: Noch jetzt nimmt eine Anzahl von Forschern an, dass es die Eizelle isť, welche die Fähigkeit besitzt, alle möglichen Gewebselemente aus sich hervorgehen zu lassen und dass demgemäss die Teratome und Dermoidcysten der Ovarien durch atypische Entwicklung unbefruchteter Eizellen zu erklären seien. Dieser Theorie steht Askanazy ablehnend gegenüber, indem er in These $V$ sagt: „Es gibt ausserhalb der Keimdrüsen Dermoide mit Produkten dreier Keimblätter und einem eigenen Nervensystem, welche wie die Dermoide im Mediastinum auf verirrte Teile der Genitaldrüsen bzw. ihrer Anlagen nicht zurückgeführt werden können." Dieser Satz hat natürlich auch für die Teratome der Orbita Gültigkeit und man wird deshalb gleichfalls jene Erklärungsweise ablehnen müssen, wenn man nicht die unwahrscheinliche Annahme machen will, dass Tumoren von ganz der gleichen Zusammensetzung auf zwei prinzipiell ganz verschiedene Arten entstehen können.

Marchand, der die eben geschilderte Erklärung ablehnte, wollte ursprünglich die Teratome von befruchteten Polzellen ableiten, welche bei der Reifung des Eies aus diesem austreten. Dieser Annahme sind andere Autoren, z. B. Fischel'2), entgegengetreten, weil sie den Polzellen ihrer ganzen Zusammensetzung nach und auf Grund experimentell-embryologischer Ergebnisse nicht die Fähigkeit zutrauen, derartige Tumoren zu bilden, und weil ferner im Ovarium gleichzeitig fünf Dermoide beobachtet sind, während es höchstens drei Polzellen gibt. Die Theorie wurde deshalb modifiziert und an die Stelle der
1) Loc. cit.
2) Loc. cit. 
Polzelle die abgesprengte Blastomere gesetzt. In dieser Form, der sich jetzt die meisten Autoren zugewandt haben, wurde sie schon von mir bei der Besprechung der Schwalbeschen Untersuchungen über den Epignathus verwertet.

Wendet man sie auf die Teratome der Ovarien an, so sind also auch diese aus Elementen herzuleiten, welche zu irgend einer Zeit der Entwicklung aus dem Zellverband zunächst ausgeschaltet und dann in denselben eingeschlossen sind $^{1}$ ).

Es ist nun gewiss interessant sich die Frage vorzulegen, warum die bisher bekannten Teratome der Orbita schon bei der Geburt als grosse Tumoren vorhanden waren, die der Ovarien aber regelmässig erst im spätern extrauterinen Leben entstehen.

Da man wegen der übereinstimmenden Zusammensetzung der Tumoren den abgesprengten Gewebskeimen annähernd gleiche Potenz zuschreiben muss, so ist es schwer sich vorzustellen, dass jenes verschiedene Verhalten darauf beruht, dass die Zeit der Isolierung jener hypothetischen Keime eine sehr verschiedene ist. Anderseits spricht manches für die Annahme, dass je früher (etwa im wirklichen Blastomerenstadium) die Absprengung erfolgt, um so weniger Neigung zum Latentbleiben des Keimes erkennbar ist. Ist doch beispielsweise die

1) Ich möchte nicht unterlassen, hier darauf hinzuweisen, dass Fischel ${ }^{1}$ ) sowohl der Polzellen - wie der Blastomerenhypothese ablehnend gegenübersteht. Er nimmt zwei ihrer Genese nach verschiedene Arten fötaler Inklusion an:

1. Diejenigen, welche auf rudimentäre, ursprünglich dem Stammindividuum einfach anliegende, aber selbständige sekundäre Embryonalanlagen, eventuell auf durch irgendwelche mechanische Momente von der einen vorhandenen Anlage partiell isolierte und sich daher auch mehr oder weniger selbständig entwickeInde Bruchstücke von Embryonalanlagen zurückzuführen sind. Dahin gehören die Fälle, in welchen sich typisch entwickelte Körperteile finden. "Solche typische wohlentwickelte Körperteile können eben nur auf Teile von Embryonalanlagen zurückgeführt werden.

2. „Die Genese der übrigen, keinerlei Differenzierung zu typischen Körpergebilden aufweisenden Formen ist auf Verschiebung und Verlagerung von Teilen der Keimblätter, bzw. örtlich begrenzte pathologische Mehrprodulition von Zellen derselben zurückzuführen."

Es will mir scheinen, als ob die Unterschiede zwischen dieser Auffassung und der Art, wie Schwalbe die Blastomerenhypothese verwendet hat, geringer sind, als man vielleicht auf den ersten Blick meinen könnte. Ich verzichte aber vollständig darauf, in diesen äusserst schwierigen Fragen selbständig Stellung zu nehmen.

1) Loc. cit. 
Entwicklung eines ganzen oder auch nur unvollständigen zweiten Fötus niemals nach der Geburt beobachtet worden, wohingegen die einfachen Mischgeschwülste sehr oft erst im spätern Leben entstehen.

Dass die Zeit der Absprengung aber doch auf keinen Fall ausschlaggebend dafïr sein kann, zu welcher Zeit des intra- oder extrauterinen Lebens die Keime ihre weitere Entwicklung beginnen, das lehrt mit Bestimmtheit der von Askanazy durchgeführte Vergleich der Dermoidcysten des Eierstocks mit den soliden Teratomen desselben Organs. Beide enthalten, wenigstens in der überwiegenden Mehrzahl der Fälle, Elemente aller drei Keimblätter in mehr oder weniger regelloser Anordnung. Beide entwickeln sich aus Keimen, die zweifellos in früher embryonaler Periode in das Organ geraten sind. Die Potenz dieser Keime muss also eine annähernd gleiche sein, denn auch die Dermoide enthalten die Elemente der drei Keimblätter meist in regelloser Verteilung und bringen es nur seltener zur Ausbildung von mehr oder weniger vollständigen Organen. Der entscheidende Unterschied zwischen beiden Geschwulstformen ist aber der, dass die Dermoide aus fertig ausgebildeten Geweben bestehen, die "so alt sind wie ihre Träger" (Askanazy), die Teratome dagegen aus embryonalen Gewebsformationen zusammengesetzt sind.

Es können also Gewebskeime, die vermutlich annähernd der gleichen Entwicklungsstufe des Individuums entstammen, ihre Entwicklung in total verschiedenen - durch Dezennien getrennten Zeiträumen beginnen. Eine Beantwortung der Frage, warum zwischen den Teratomen der Orbita und Munahöhle einerseits, denen der Ovarien anderseits diese grosse Verschiedenheit besteht, lässt sich also zurzeit wohl nicht finden.

Die engen Beziehungen, welche zwischen den Dermoidcysten des Ovariums und den Teratomen desselben bestehen, könnten zu einer Betrachtung anregen, ob nicht ähnliche Verhältnisse auch an den Dermoidcysten und den Teratomen der Orbita nachgewiesen werden können.

Die Dermoidcysten der Orbita bestehen ebenso. wie die des Ovariums aus fertigen Geweben, von denen man auch annehmen kann, dass sie etwa so alt sind wie ihr Träger. Ein Unterschied scheint aber nach den bisherigen Angaben darin zu-liegen, dass ihre Wand fast immer nur aus den Elementen der Haut zusammengesetzt ist, was zu der Annahme geführt hat, dass sie in einem gewissen Stadium der embryonalen Entwicklung durch Einstülpung und Abschnürung derselben entstehen. Es scheint aber auch Ausnahmen zu geben, 
wenigstens finde ich die Angabe ${ }^{1}$ ), dass ein zellreiches Gewebe mit typischen Ganglienzellen beobachtet worden ist. Erwähnenswert ist auch, dass Levinsobn ${ }^{2}$ ) an einem auf der Hinterfläche eines kolobomatösen Auges sitzenden Dermoid kleine Knoten von Ganglienzellen und im Epithel zahlreiche Becherzellen gefunden hat. Vielleicht würde eine systematische Untersuchung an Serienschnitten manchmal einen komplizierteren Aufbau der Wand, die Beteiligung aller drei Keimblätter an demselben erkennen lassen. In diesem Falle wäre die Analogie mit den Dermoidcysten des Ovariums eine vollständige und man würde dann wohl Veranlassung haben, die Entstehung dieser Gebilde in eine frühere embryonale Periode zu verlegen, als wenn man sie aus einer Einstïlpung und Abschnürung der Haut hervorgehen lassen kann. Zurzeit ist über diese Dinge wohl nichts sicheres auszusagen; dass aber die wirklich methodische objektive Untersuchung hier unter Umständen zu veränderten Auffassungen führen kann, zeigt z. B. die Arbeit von Nobbe ${ }^{3}$ ), der in allen in Serie geschnittenen sogenannten subconjunctivalen Lipomen die Flemente der Haut nachweisen und die Geschwülste demnach als Lipodermoide bezeichnen konnte.

Jedenfalls lohnt es sich, diesen Verhältnissen erhöhte Aufmerksamkeit zuzuwenden.

\section{Anophthalmus congenitus bilateralis mit Encephalocele orbitae.}

(Hierzu Fig. 9 auf Tafel II.)

Vor sechs Jahren erhielt ich durch die Güte von Herrn Kollegen Schlodtmann, damals Assistent meines Vaters in Halle, die Präparate des folgenden Falles:

„Männlicher Fötus am 13. XI. 99 perforiert und extrahiert. Alter wahrscheinlich 12 Monate. Descensus testieulorum noeh nicht erfolgt. $\mathrm{Hy}$ drocephalus. Wolfsrachen und Hasenscharte. An beiden Händen fehlt der Daumen, an der reehten sitzt an seiner Stelle eine Bluteyste, die an einem dünnen Stiel hängt.

Die Bulbi sind änsserst rudimentär, sie wurden mit der Sella tureica und dem darin befindlichen Chiasma im Zusammenhang herausgenommen.

3) v. Duyse, Eléments d'Embryologie et de Tératologie de l'oeil. S. 409.

2) Levinsohn, Kurzer Beitrag zur Histologie angeborener Augenanomalien. v. Graefe's Arch. f. Ophthalm. Bd. LVII. S. 266.

3) Nobbe, Über die Lipodermoide der Conjunctiva. v. Graefe's Arch. í. Ophthalm. Bd, XLIV. 3.

จ. Graefe's Archiv für Ophthalmologie. LXIII. 1. 
Das linke Auge, das nur ein formloser Gewebsfetzen ist, wurde bei der Präparation leider abgetrennt. Die Präparate wurden in Müllersche Flüssigkeit gelegt."

Ich habe hier den Wortlaut der mir zugegangenen Mitteilung wiedergegeben, die anatomische Untersuchung wird zeigen, dass die auf Grund der makroskopischen Betrachtung gemachten Angaben nicht zutreffend sind.

Das Gebilde, welches für den rechten Bulbus gehalten wurde, hat einen Durchmesser von 10-11 mm, annähernd kuglige Gestalt und setzt sich nach hinten in einen Stiel fort. Von einer Cornea ist nichts zu sehen. Celloidineinbettung und Schnittserie in frontaler Richtung.

Das dem linken Bulbus entsprechende Gebilde hat mehr unregelmässig längliche Gestalt: grösste Länge 10, grösster Dickendurchmesser $5 \frac{1}{2} \mathrm{~mm}$. Auch dieses wird in Serienschnitte zerlegt, deren Richtung aber nicht genauer angegeben werden kann.

Das Stück der Schädelbasis wird entkalkt, in toto eingebettet und frontal geschnitten.

Als wichtigstes Ergebnis kann der Satz vorangestellt werden, dassdie bëiden für Bulbusrudimente gehaltenen Gebildeausschliesslich aus nervöser Substanz bestehen, und dass in dem ganzen übersandten Materiale von Bulbusrudimenten keine Spur nachweisbar ist. Wenigstens fehlen Elemente der Augenblase vollkommen, nur in ein paar Schnitten trifft man innerhalb der den Pseudobulbus umhüllenden Membranen einige pigmentierte Zellen; dieselben haben aber nicht den Charakter von Pigmentepithel, sondern eher von chorioidealen Stromazellen mit ihren charakteristischen. Ausläufern. Es ist also nicht ausgeschlossen, dass hier ein kleiner

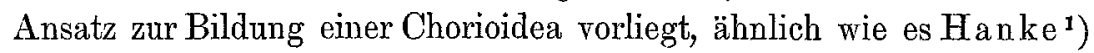
in seinem Falle gefunden hat: die Hauptsache bleibt der absolute Mangel eines als Augenblase oder als Rest einer solchen zu deutenden Gewebsteiles.

Die mikroskopische Untersuchung ergibt folgende Einzelheiten:

Beiderseits zeigt das als Bulbus angesprochene Gebilde einen Überzug von einer sehr gefässreichen bindegewebigen Kapsel, welche Ähnlichkeit mit der Pia hat, ohne dass man berechtigt wäre, sie mit voller Bestimmtheit als weiche Hirnhaut anzusprechen.

Ein Schnitt durch den rechten Pseudobulbus lässt bei Hämatoxylin-

1) Hanke, Zwei seitene Missbildungen des Bulbus. v. Graefe's Arch. f. Ophthalm. Bd. LVII. S. $28 \mathrm{ff}$. 
Eosinfärbung eine hellere zentrale von einer dunklern peripheren Zone unterscheiden, eine scharfe Grenze existiert aber nicht. Die Farbendifferenz beruht auf verschiedenem Zellreichtum.

In einer äusserst zart retikulierten Grundsubstanz finden sich Zellen von sehr verschiedener Art eingelagert: 1. kleine mit spärlichem Protoplasma und einem runden, intensiv gefärbten Kern. 2. Grössere mit einem rundlichen oder ovalen lichtern Kern, der mehrere Kernkörperchen enthält; eine sichere Deutung dieser beiden Zellarten ist nicht möglich. Mit Wahrscheinlichkeit sind sie für Gliazellen anzusehen. 3. Sehr grosse mit Fortsätzen versehene Zellen, die nach dem Urteil von Herrn Prof. Nissl, der die Liebenswürdigkeit hatte, die Präparate anzusehen, als sichere Ganglienzellen zu betrachten sind. Die Entscheidung, welche bei dem Mangel elektiver Färbung sehr schwierig ist, stiitzt sich auf folgende Punkte: Die Grösse der Zellen wäre für Gliazellen sehr angewöhnlich, in den grossen Gliazellen pflegt aber der Kern exzentrisch zu liegen, was hier nicht der Fall ist. Die Begrenzung des Zellkernes ist hier eine buchtige, was bei MüllerPräparaten an den Ganglienzellen gewöhnlich ist, bei den Gliazellen nicht vorzukommen pflegt. An den Kernkörperchen erkennt man die von Nissl so genannten Polkörper, endlich sind ausgesprochene Faltungen der Kernmembran zu sehen, beides Befunde, die für Ganglienzellen charakteristisch sind.

Sichere Nervenfasern konnten nicht nachgewiesen werden. Die Weigertsche Färbung schwärzt zwar gewisse Bezirke mehr oder weniger stark, die Ölimmersion zeigt aber nur feine schwarze Pünktchen. Ob dieselben Niederschläge darstellen, erscheint mit Räcksicht auf gewisse Eigentümlichkeiten ihrer Verteilung zwar zweifelhaft, jedenfalls aber ist der Befund nach Nissls Urteil nicht genügend für den Nachweis von Nervenfasern.

Die zarte retikulierte Substanz tritt an einzelnen Stellen in Form breiterer äusserst zellarmer Streifen auf und sieht an diesen Stellen wie Gliagewebe aus, an andern Stellen hat ihre Anordnung gar nichts Charakteristisches. Wichtig ist, dass sie fast überall, wo der bindegewebige (piale?) Überzug an den Pseudobulbus grenzt, einen schmalen zellarmen Streifen bildet, ein Befund, der nach Nissls Mitteilung überall am Zentralnervensystem gefunden wird. Nissl glaubt mit grosser Wahrscheinlichkeit Gliasubstanz annehmen zu dürfen. Nachträglich habe ich noch in Schnitten, die mit Heidenhains Hämatoxylia gefärbt sind, deutliche gefärbte Fasern sehen können, welche senkrecht zur Oberfläche des Tumors verlaufen und sich mit verbreiterten Enden (Gliafüsse?) an die Gefässwand ansetzen.

Das der linken Orbita angehörige Gebilde zeigt ähnliche Befunde wie das rechte, nur fand ich dort keine Ganglienzellen, dagegen ist die Anordnung des Gliafaserfilzes als zellarmer Streifen dort viel deutlicher als rechts (Fig. 9).

Bemerkenswert ist hier noch das Auftreten massenhafter Kalkkonkremente, die sich mit Hämatoxylin schwarzblau färben. Auf der rechten Seite kommen nur vereinzelte vor. Beiderseits finden sich ausserordentlich zahlreiche Gefässo sowie Blutungen. In den Gefässen der bindegewebigen Kapsel sieht man stellenweise reichliche Kalkeinlagerungen in die Media derart, dass entweder das ganze Gefäss einen starren Ring bildet, oder an 
einzelnen Stellen Buckel in das Lumen vorspringen. Die Intima ist dabei überall erhalten.

Der Stiel des rundlichen Gebildes, der bei der Sektion für den Nervus opticus angesehen war, entspricht diesem histologisch in keiner Weise, sondern er besteht aus demselben Gewebe wie der grösste Teil des Pseudobulbus. Ganglienzellen finden sich in ihm nicht. Ganz aus demselben Gewebe ist auch der für ein Chiasma gehaltene Teil des Präparates zusammengesetzt, wie Frontalschnitte durch die entkalkte Schädelbasis zeigen.

In den durch den Stiel gelegten Schnitten findet man in unmittelbarer Nachbarschaft desselben den Querschnitt einer sehr starken Arterie, welche als Arteria ophthalmica angesprochen werden darf.

\section{Epikrise.}

Bei einem wahrscheinlich abnorm lange getragenen Fötus wurde wegen Hydrocephalus die Perforation und Extraktion gemacht. Ausser einigen andern Missbildungen, auf welche noch kurz einzugehen sein wird, fanden sich in beiden Orbitae kuglige Gebilde, welche bei der Sektion für rudimentäre Bulbi gehalten wurden, um so mehr, als sie durch einen Stiel, den man für den Nervus opticus ansah, mit dem Gehirn in Verbindung standen. Es ist wohl kaum zu bezweifeln, dass derselbe das Foramen opticum durchsetzt hat, sonst wäre er wohl nicht für den Nervus opticus gehalten worden.

Die mikroskopische Untersuchung hat nun aber ergeben, dass die kugligen Gebilde, welche für rudimentäre Bulbi gehalten waren, mit solchen nicht das geringste zu tun haben, sondern dass sie aus nervöser Substanz bestehen.

Von Augäpfeln ist in dem untersuchten Material nichts nachzuweisen, wenigstens gilt dies mit Sicherheit für die Derivate der Augenblase und die Linse. $\mathrm{Ob}$ die paar pigmentierten verästelten Zellen vom Charakter der chorioidealen Stromazellen wirklich einer rudimentären Chorioidea angchören, mag dahingestellt bleiben.

Der sichere Nachweis von Ganglienzellen und der mit hoher Wahrscheinlichkeit erbrachte von Gliagewebe berechtigen dazu, die für Bulbi gehaltenen Gebilde als aus nervöser Substanz zusammengesetzt anzusehen. Wenn sie auch mit irgend einem normalen Hirnteil keinerlei Ähnlichkeit aufweisen, so wird man sie doch als atypisch differenzierte Gehirnsubstanz betrachten dürfen. Da sie durch einen aus derselben Substanz gebildeten Stiel mit dem Gehirn in Zusammenhang stehen und einen Überzug von einer der Pia ähnlichen Haut besitzen, so darf man sie - rein topographisch betrachtet mit einigem Rechte als Meningo-Encephalocelen bezeichnen. 
Es entsteht nun die Frage: wie ist ihre Entstehung aufzufassen? Wie bei fast allen Missbildungen ist hier eine bestimmte Antwort unmöglich, man kann aber doch folgende Uberlegung anstellen:

In dem untersuchten Material ist von Elementen der Augenblase sowie von Sehnerv und Chiasma keine Spur nachzuweisen. Leider stand nicht der ganze Orbitalinhalt zur Verfügung, es kann daher nicht mit absoluter Sicherheit ausgeschlossen werden, dass doch ein Bulbusrudiment vorhanden war. Immerhin ist dies unwahrscheinlich.

Nimmt man an, dass Bulbusrudimente fehlten, und berïcksichtigt, dass der Stiel der Pseudobulbi jedenfalls durch das Foramen opticum ging (sonst hätte man ihn wohl nicht für den Nervus opticus gehalten), so liegt folgender Gedanke nahe: Nach Ausstülpung der primären Augenblasen ist es nicht zur Bildung der sekundären gekommen, sondern die primären mit dem Augenblasenstiel haben sich zu atypischer nervöser Substanz weiter entwickelt.

Selbstrerständlich bleibt diese Vorstellung eine Hypothese, beweisbar ist sie nicht. Nimmt man sie aber an, so erkennt man, dass die in den Augenhöhlen befindlichen Gebilde dann nicht MeningoEncephalocelen im gewöhnlichen Wortsinn darstellen.

Ein eigentlicher Hirnbruch, der durch das Foramen opticum in die Augenhöhle dringt, ist meines Wissens bisher nicht beobachtet worden, ebensowenig aber die Kombination von Meningo-Encephalocele und Anophthalmus congenitus. Der Fall ist demnach wohl als einzig in seiner Art zu betrachten.

Noch am meisten Ähnlichkeit zeigt er mit einer Beobachtung von de Waele und Lew ouillon ${ }^{1}$ ): Dieselben fanden bei einem einjăhhrigen Kinde einen scheinbaren Anophthalmus der linken Seite kombiniert mit einem orbitalen Tumor und doppelter Spaltung des obern Lides. Die histologische Untersuchung des exstirpierten Tumors ergab das Vorhandensein eines rudimentären Bulbus, es wurde chorioideales Pigment, retinales Pigmentepithel sowie embryonale Retina und das Rudiment einer Linse(?) gefunden. Der Tumor wird als Glioma cerebroides bezeichnet. Ein Vergleich der histologischen Beschaffenheit mit meinem Falle ist wegen der Mangelhaftigkeit der Abbildung schwierig. Die Beziehung des Tumors zum Gehirn konnte nicht

1) de Waele und Lewouillon, Colobome de la paupière supérieure et gliome cérébroide de l'orbite. Ann. d'Ocul. Tom. CXXV. p. 321. 
aufgeklärt werden, ebensowenig wie in dem von Krückmann ${ }^{1}$ ) als Meningo-Encephalocele des Bulbus beschriebenen merkwürdigen Falle. $\mathrm{Da}$ in diesen beiden einmal ein recht gut ausgebildeter, das andere Mal ein zwar rudimentärer, aber doch beide Blätter der sekundären Augenblase enthaltender Bulbus vorhanden war, so ist natürlich der Erklärungsversuch, den ich für meinen Fall gegeben habe, auf jene nicht anwendbar.

$\mathrm{Zu}$ gesicherten Ergebnissen kann man aber bei einem Vergleich dieser drei Fälle leider nicht kommen, weil bei meinem nicht der ganze Orbitalinhalt untersucht wurde, bei jenen die Verbindung des orbitalen Tumors mit dem Gehirn unaufgeklärt bleiben musste.

Natürlich ist bei meinem Falle auch mit der Möglichkeit zu rechnen, dass eine Augenanlage in den frühesten Entwicklungsstadien durch irgend welche Einflüsse zu Grunde gegangen ist, und dass die gefundenen Encephalocelen den in den beiden andern Beobachtungen beschriebenen gleich zu setzen sind.

De Waele und Lewouillon führen die Missbildung in ihrem Falle auf das Amnion zurück und begründen diese Annahme mit vollem Recht dadurch, dass das obere Lid eine doppelte Einkerbung zeigte, dass der Canthus externus fehlte, die Conjunctiva hier vielmehr ganz allmählich ins Niveau der äussern Haut überging, ferner damit, dass an der vordern Wand des linken Nasenlochs eine Hautpapille und auf der linken Wange, Lippe sowie am Kinn warzenähnliche Hervorragungen bestanden.

Dass auch für meinen Fall amniogene Störungen in Erwägung gezogen werden müssen, geht aus dem Vorhandensein von Hydrocephalus, Wolfsrachen und Hasenscharte sowie dem Fehlen beider Daumen hervor. Letzterer Befund ist wohl als Amputation durch Amnionstränge aufzufassen, $d a$ an Stelle des rechten Daumens eine Blutcyste gefunden wurde, die an einem dünnen Stiel hing.

Man könnte sonach auch an ein Zugrundegehen der Augenanlagen in frühesten Stadien durch Amniondruck denken und hätte dann allerdings keine Erklärung für die Bildung der Encephalocelen.

Ich begnüge mich mit diesen kurzen Erwägungen und versenke mich nicht weiter in Hypothesen, die die Erkenntnis nicht wesentlich fördern dürften.

Nur muss ich zum Schluss noch in Kürze auf die Frage nach

1) Krü ckmann, Über eine Meningo-encephalocele des Augapfels. v. Gra ef e's Arch. f. Ophthalm. Bd. XLVII. 
der Entstehung und Bedeutung des Anophthalmus congenitus im allgemeinen eingehen, weil ich hier den Standpunkt, den ich in zwei frühern Arbeiten ${ }^{1}$ ) vertreten habe, nicht mehr in vollem Umfang aufrecht erhalten kann. Es geht mir in der Beziehung ähnlich, wie gegenüber dem Colobom.

Im Graefe-Saemisch habe ich die Ansicht ausgesprochen, dass in sehr seltenen Fällen die Ausstülpung der Augenblase überhaupt unterbleiben mag, dass es sich aber beim Anophthalmus meistens um die Zerstörung der Augenblase durch fötale Krankheiten handelt; ich hatte in dieser Hinsicht auf das Vorhandensein eitriger Conjunctivitis beim Neugeborenen noch erheblichen Wert gelegt und angenommen, dass in diesen Fällen die eitrige Zerstörung der Augenanlage in einem relativ späten Entwicklungsstadium erfolgt sei. Als „sehr wohl denkbar" hatte ich es schliesslich bezeichnet, dass abnorme Enge des Amnion zur Zerstörung des Auges durch Druck führen könnte.

Eine Anzahl neuer anatomischer Befunde und meine eigenen embryologischen Untersuchungen über das Colobom veranlassen mich dazu, die Ansicht, dass es sich beim Anophthalmus in der Mehrzahl der Fälle um eine Phthisis bulbi höchsten Grades infolge fötaler Entzündung handle, aufzugeben. So wenig ich daran zweifle, dass fötale Entzündungen, die zu Phthisis bulbi führen, tatsächlich vorkommen, so ist doch daran festzuhalten, dass dies Erkrankungen in vorgerückteren Entwicklungsstadien sind, während wir Veranlassung haben, die Entstehung des Anophthalmus in die friihesten Zeiten des Fötallebens zu verlegen. Ich erkenne jetzt auch die Berechtigung des Einwandes von Hoppe ${ }^{2}$ ) an, der eine bei der Geburt gefundene Eiterung der Conjunctiva nicht mit der Entstehung des Anophthalmus in Zusammenhang bringen will. Für die frühe Entstehung des Anophthalmus spricht unbedingt, dass jetzt mehrere zuverlässige Untersuchungen vorliegen, welche das völlige Fehlen jeder Spur von Elementen der sekundären Augenblase beweisen. Dies ist nur möglich, wenn sie entweder gar nicht gebildet oder im allerfrühesten Stadium zu Grunde gegangen ist. Dass ihre Bildung ausbleiben kann, ist durch Hess ${ }^{3}$ ) für das Hühnchen, durch

1) E. v. Hippel, Über Anophthalmus congenitus. v. Graefe's Arch. f. Ophthalm. Bd. XLVII. S. 227 und Die Missbildungen und angeborenen Fehler des Auges in Graefe-Saemisch Handb. d. ges. Augenheilk. 2. Aufl.

2) Hoppe, Fötale eitrige Entzündung als Ursache des congen. Mikrophth. u. Anophthalmus. Arch. f. Augenheilk. Bd. XXXIX. 3. S. 201.

$\left.{ }^{3}\right) \mathrm{Hess}$, Beitr. zur Kenntnis der pathol. Anatomie der angeborenen Missbildungen, v. Graefe's Arch. f. Ophthalm. Bd. XXXVIII. 3. S. 18. 
Fis chl1) auch für den Menschen bewiesen. In letzterem Falle handelt es sich allerdings um einen auch sonst völlig abnormen Embryo. Ich habe früher die Meinung ausgesprochen, dass in den Fällen, wo gar keine Augenanlage vorhanden ist, auch die Augenhöhle mit ibren Bestandteilen und die Lider sich nicht bilden würden, und Greeff ${ }^{2}$ ) stimmt dieser Auffassung zu. Tch habe aber auch schon damals betont, dass die Situation im wesentlichen dieselbe ist, wenn die Angenanlage überhaupt nicht gebildet wird, oder wenn sie wieder zu Grunde gegangen ist, ehe die Bildung von Augenhöhle und Lidern begonnen hat.

Es scheint mir jetzt aber doch zweifelhaft, ob man berechtigt ist, eine solche Abhängigkeit der Entwicklung der Nebenorgane von der des Bulbus anzunehmen, vielmehr scheinen mir Fälle wie der von Hanke direkt zu beweisen, dass Schutz- und Nebenorgane des Auges sich unabhängig von der Bildung einer Augenanlage differenzieren und weiter entwickeln können. Im gleichen Sinne spricht auch der neueste Fall, den Ovio $\left.{ }^{3}\right)$ kuirzlich mitgeteilt hat: absoluter Mangel der Bulbi, Vorhandensein der Tider, der Orbita und der Muskulatur. Ich möchte mich deshalb den Ausführungen Hankes durchaus anschliessen und verweise betreffs der neuern Literatur auf seine Arbeit.

Die nahe Beziehung gewisser Fälle von scheinbarem Anophthalmus zum Mikrophthalmus weist ferner darauf hin, dass diese Gruppe durch eine Störung zur Zeit des Schlusses der Fötalspalte entstehen kann in einer Art, wie ich es in meiner Arbeit über das Colobom angedeutet habe. Dass sogenannte „innere Ursacben" in der Ätiologie des Anophthalmus eine Rolle spielen, beweist das festgestellte Ausbleiben der Augenanlage, ferner der Nachweis hereditärer Beziehungen in gewissen Fällen und die Beziehung des Anophthalmus zu Mikrophthalmus und Colobom.

Es ist aber sehr wohl möglich, dass auch der Einfluss des Amnion für die Entstehung zahlreicher Fälle von Bedeutung ist, wie Greeff dies annimmt. Man wird die einzelnen Fälle daraufhin zu untersuchen haben, und es ist kein Zweifel, dass diese Erklärungsweise für den Fall des Autors die wahrscheinlichste ist. Auch für meinen kann sie in Betracht gezogen werden, und ich werde in einem

1) Fischl, Über einen sehr jungen menschlichen Embryo. Zeitschr. f. Heilkunde. Bd. XXIV. Heft 1. 1903.

2) Greeff, Über Anophthalmus usw. Arch. f. Augenheilk. Bd. L. 1. S. 1.

3) Ovio, Caso di Anoftalmo bilaterale. La Clinica oculist. 1905. Ottobre. 
folgenden Abschnitt einen weitern beschreiben, wo ein rudimentärer Bulbus von einem grossen Dermoid umwachsen ist und noch andere Anomalien für die Bedeutung der Einwirkung des Amnions sprechen.

Ich möchte meinen jetzigen Standpunkt dahin zusammenfassen, dass uns für eine einheitliche Erklärung der Genese aller Fälle von Anophthalmus noch die genügenden tatsächlichen Unterlagen fehlen, dass wahrscheinlich verschiedene Ursachen zum klinischen Bilde des Anophthalmus führen können, dass aber die fötale eitrige Entzündung aus der Reihe der ätiologischen Momente zu streichen ist. Damit ist nicht gesagt, dass nicht gewisse Fälle hochgradiger fötaler Phthisis bulbi im klinischen Bilde erhebliche Ähnlichkeit mit dem Anophthalmus darbieten können.

\section{Kryptophthalmus congenitus.}

Den folgenden Fall habe ich in einem kurzen Autoreferat ${ }^{1}$ ) über einen im hiesigen medizinischen Verein gehaltenen Vortrag bereits mitgeteilt. Wenn ich ihn hier noch einmal erwähne, so geschieht es, weil es mir auch bei dieser Missbildung im höchsten Grade zweifelhaft geworden ist, ob die bisher allgemein gültige Erklärungsweise (fötale Entzündung mit Obliteration des Conjunctivalsacks) aufrecht erhalten werden kann.

Ich gebe zunächst die Krankengeschichte des Falles, die ich zum Teil der Liebenswürdigkeit von Herrn Dr. Würzburger in Rappenau verdanke.

Anamnese: Die Sehwester der Grossmutter des Kindes war eine Zwergin, die auf Jahrmärkten gezeigt wurde, weil sie auf einem Teller tanzen konnte. Sonst ist von Missbildungen in der Ascendenz nichts bekannt. Vor sechs Jahren hat der behandelnde Kollege die Mutter des Kindes bereits einmal von einem Monstrum entbunden, worüber er mir folgendes mitteilt: „Ich wurde gerufen, als der Kopf des Kindes geboren war und die Geburt nicht weiter vonstatten ging. Als ieh kam, war der cyanotiseh aufgedunsene Kopf vor der Scheide, Carotispuls fehlte. Vorsichtige Extraktionsversuche ohne Erfolg. Bei hoher Untersuchung im Becken eine fluktuierende Gesehwulst als Geburtshindernis, die ich als fötalen Ascites erkannte. Ich eröfnete denselben intrauterin mit der langen Schere unter Leitung der Hand, es ergoss sich eine Unmasse von Flüssigkeit, worauf die Extraktion am Kopf leicht gelang. Ich bemerkte in meinem

1) E. v. Hippel, Demonstration eines Falles von Kryptophthalmus. (Naturhist. med. Verein Heidelberg.) Münch. med. Wochenschr. 1904. S. 1575. 
Tagebuch: beideAugen als Furche angelegt, Schwimmhautbildung der Finger, beiderseits Genu varum und Pes varus. Ohrmuseheln rudimentär, Zwischenkiefer deutlich sichtbar. Ob eine Hasenscharte vorlag, ist mir nicht mehr erinnerlich."

Das am 3. IV. 04 geborene, bei der Aufnahme 10 Tage alte Kind ist gut entwickelt, Länge $50 \mathrm{~cm}$, trinkt gut, Stuhl und Urin normal.

Schädel etwas quadratisch, Umfang $35 \mathrm{~cm}$. Fontanellen in normaler Weise zu füblen. Ziemlich reichliche Behaarung, auch auf der Stirne; links setzt sich ein etwas dichterer Streifen von Haaren von der Stirne nach der Gegend des innern Augenwinkels fort, weniger ausgesprochen ist diese Erscheinung rechts, wo der Streifen mehr nach aussen gerichtet ist.

Der Nasenrücken liegt sehr tief und ist ausserordentlich breit. Auf der rechten Seite zieht die Haut kontinuierlich über die Gegend des Auges und des innern Winkels fort. Die Stelle der Lidspalte ist durch einen schmalen, etwas hellern Streifen angedeutet, der nicht wie eine Narbe aussieht, beim Schreien zieht sich diese Stelle als deutliche Längsfurche ein. Am temporalen Ende des Streifens ist eine kleine grubige Einziehung dauernd vorhanden. Dieselbe ist mit reichlichen Lanugohärchen besetzt. Die Angengegend ist halbkuglig vorgewölbt und man sieht ganz deutliche Bewegungen des Bulbus unter der Haut verlanfen. Der deutlich fühlbare Bulbus ist leidlich gespannt, fühlt sich kuglig an und scheint kleiner als ein normales Auge zu sein.

Links zieht die Haut ebenfalls über die Augengegend hinweg bis auf einen kleinen temporalen Bezirk, von dem gleich zu sprechen sein wird. An ihr ist absolut nichts von einer Raphe zu bemerken, auch beim Schreien nicht, das Hautstück hat vielmehr vollkommen gleichmässige Beschaffenheit. Im temporalen Teil ist ein Stück Lidspalte von dreieckiger Form erhalten, oberes und unteres Lid sind auf eine Strecke von etwa $3 \mathrm{~mm}$ vorhanden, wenn auch sehr niedrig, ihr Rand ist mit normalen Cilien besetzt und beim Auseinanderziehen der Lider kommt man in einen deutlichen Conjunctivalsack; ob sich derselbe von hier aus nasal fortsetzt, ist zunächst nicht zu entscheiden. Der Bulbus macht deutliche Bewegungen und erscheint für die Palpation grösser wie der rechte.

Bei stärkerem Lichteinfall ist keine Runzelung der Haut, ebensowenig eine besondere Lebhaftigkeit der Augenbewegungen zu bemerken, der Nachweis, dass Lichtempfindung besteht, ist demnach nicht erbracht.

Sonstige Anomalien finden sich an den Extremitäten und den Genitalien.

Rechte Hand: Daumen und Zeigefinger sind frei, die drei andern Finger zeigen ausgesprochene Syndactylie, nur die Endphalangen sind frei.

Linke Hand: Syndactylie zwischen allen Fingern, der dritte und vierte zeigen totale Verwachsung, an der sogar die Nägel beteiligt sind. Bei den übrigen Fingern ist die Endphalanx frei.

Rechter Fuss: Syndactylie zwischen dritter, vierter und fünfter Zehe.

Linker Fuss: Syndactylie zwischen vierter und fünfter Zehe.

Scrotum und Hoden normal. Glans Ponis eigentümlich posthorn- 
förmig nach rechts gekrümmt, Präputium ganz mangelhaft entwickelt, die Spitze der Glans ragt ziemlich weit daraus hervor.

Die Insertion der Nabelschnur liegt abnorm tief, der Nabel zeigt leichte Entzündung, die in einigen Tagen ausheilt.

Untersuchung in Narkose zeigt, dass man mit der Sonde von dem kleinen Conjunctivalsack aus ein kleines Stückehen unter der Haut in nasaler Richtung vordringen kann. Die Haut wird hier auf der Hohlsonde gespalten, die Wundränder mit dem Conjunctivalrand umsäumt, wodurch ein Conjunctivalsack von im ganzen etwa $1 \mathrm{~cm}$ Länge entsteht.

Am 29. VI. 04 wird rechts der Versuch einer Operation zur Freilegung des Bulbus gemacht (Prof. Leber). Langer horizontaler Schnitt der Lage der fehlenden Lidspalte entsprechend. Nach Spaltung einer ziemlich dicken Hautschicht kommt in der Tiefe ein kleiner, kaum haselnussgrosser, weicher, bläulich schimmernder Körper zum Vorschein. Die über ihm liegende feine Membran wird an einer kleinen Stelle angeritzt, es tritt ein Tröpfehen klarer Flüssigkeit aus. Von einem Conjunctivalsack ist nichts zu finden, auf ein Freipräparieren des Bulbus wird verzichtet, da ein sehtïchtiges Auge auszuschliessen ist.

Die Beschreibung der Operation lässt erkennen, dass eine Orientierung über die topographischen Verhältnisse und die genauere Beschaffenheit des Bulbus nicht möglich war. Dass der ganze Bulbus nur die Grösse eines Haselnusskerns gehabt haben soll, halte ich nach dem Ergebnis der Palpation für ausgeschlossen.

Von weitern therapeutischen Versuchen wird abgesehen. Seit meiner zusammenfassenden Darstellung sind noch folgende Fälle von Kryptophthalmus veröffentlicht worden:

1. Blessig ${ }^{1}$ ). Zweijähriges Kind. Rechts unteres Lid normal, oberes vollständig mit der Bulbusoberfläche verwachsen. Bulbus ungefähr von normaler Grösse. Links: Haut vollständig über die Augengegend ziehend, darunter eine weich-elastische Geschwulst, im nasalen Teil der Augenhöhle ein rundlicher Körper fühlbar. Keine andern Missbildungen.

Links Operation: es zeigte sich dabei, dass die weich-elastische Geschwulst nur aus Fettgewebe bestand, dass der rundliche Körper dem erweiterten Conjunctivalsack entsprach und dass der Bulbus selbst so tief lag, dass man ihn nicht fühlen konnte. Er hatte eine klare, grosse und stark gewölbte Hornhaut, die Iris schien zu fehlen, man bekam rotes Licht. Im weitern Verlauf erfolgte eine unaufhaltsame Verkleinerung der angelegten Lidspalte dadurch, dass die Wände des Conjunctivalsackes, welcher eine zähe Masse absonderte, miteinander verklebten, schliesslich entstand eine ganz schmale Fistel, Patient konnte nicht weiter beobachtet werden.

Blessigs Auffassung geht dahin, dass es sich beim Kryptophthalmus nicht um einen Bildungsmangel der Lider, sondern in den Fällen mit Conjunctivalsack um eine Verwachsung der Lidspalte,

1) Blessig, Fall einer seltenen Missbildung des Auges, Klin. Monatsbl. f. Augenheilk. 1900. S. 652 . 
in denen ohne Conjunctivalsack um eine fächenhafte Verwachsung der Lider mit dem Bulbus und untereinander handelt, die Entstehungszeit sei in den zweiten bis dritten Monat des embryonalen Lebens $\mathrm{zu}$ verlegen. Über die eigentlichen ätiologischen Momente liessen sich wohl nur Vermutungen anstellen. Was die oft hochgradigen Veränderungen des Bulbus selbst anbetreffe, so sei ihr Zusammenhang mit der Lidanomalie noch nicht aufgeklärt.

Ich möchte mit Golowin $\left.{ }^{1}\right) Z_{\text {weifel }}$ äussern, ob der bei der Operation von Blessig festgestellte objektive Befund sicher zutreffend gedeutet worden ist. Blessig schreibt: „Nach Eröffnung des (Conjunctival-) Sackes durch einen Horizontalschnitt fliesst klare Flüssigkeit ab und präsentiert sich ein enger, sehr tiefer Schleimhautsack, an dessen Grunde, in einer Tiefe von mehrern Zentimetern, ein sphärisches Gebilde mit durchsichtigem vordern Pol sichtbar wird. Beim Hineinleuchten mit der Fuchsschen elektrischen Kugellampe in die Tiefe des Sackes leuchtet der Hintergrund dieses als Bulbus mit klarer Cornea anzusprechenden Gebildes im roten Lichte eines normalen Augenhintergrundes auf. Der Bulbus ist nur wenig kleiner als der rechte, die Cornea ist gross und auffallend stark gewölbt, die Iris scheint zu fehlen."

Nach dieser Beschreibung und der Angabe, dass der gebildete "Conjunctivalsack" durch Ausscheidung einer zähflüssigen Masse wieder verklebte und sich unaufhaltsam verkleinerte, scheint es mir wie Golowin sehr wohl möglich, dass der Raum, welcher als Conjunctivalsack angesehen wurde, der cystisch erweiterten vordern Kammer, und die scheinbar klare Hornhaut der etwas verdichteten Glaskörperoberfläche entsprochen hat. Bei der sehr tiefen Lage des Bulbus wird eine ganz sichere Beurteilung der Verhältnisse wohl äusserst schwierig gewesen sein.

Golowin hat zwei Fälle mitgeteilt.

I. 26 jähriger Bauer: Rechts totales Eetropium des untern Lides. Von oben her' zieht die Haut auf den Bulbus und ist mit seiner Oberfläche vollständig verwachsen. Knorpel und Cilien fehlen. Bulbus ist etwa haselnussgross. Links zieht die Haut von der Stirne ununterbrochen auf die Wange hinüber, im temporalen Teil eine flache Vertiefung mit einigen Härchen. Walnussgrosser Bulbus. Lichtempfindung nicht nachzuweisen. Keine Heredität, keine andern Missbildungen. Bei einer Operation, die auf Wunseh des Patienten zu kosmetischen Zweeken gemacht wurde, fand sich

1) Golowin, Beiträge zur Anatomie und Pathogenese des hryptophthalmus congenitus. Zeitschr. f. Augenheilk. Bd. VIII. S. 175. 
ein kleiner knorpelartiger Bulbus, keine Hornhant, kein Conjunctivalsack. Der Bulbus mit der bedeckenden Haut wurde entfernt.

II. Zwei Monate altes Kind. Beiderseits zieht die Haut ununterbrochen von der Stirne über die Bulbusgegend auf die Wange hinüber. Augäpfel ungefähr von normaler Grösse fühlbar. Links deutliche Lichtemptindung. Bei einem Operationsversuch musste die Haut von der Bulbusoberfäche abpräpariert werden, die Bulbusoberfäche hatte das Aussehen eines Hornhautstaphyloms; der Versuch, die Lider plastisch herzustellen, misslang. Eine Schwester des Kindes hat die gleiche Anomalie.

Andogskyl) bat noch drei Fälle von Kryptophthalmus beobachtet. Nach dem kurzen Referat von Natanson, das mir allein zugänglich ist, wurden in dem einen Falle nach Durchtrennung der Hautdecke etwas verkleinerte Bulbi mit trüber skleraähnlicher Cornea gefunden, in den beiden andern war der Kryptophthalmus der einen Seite mit Mikrophthalmus der andern vergesellschaftet.

Goldzieher ${ }^{2}$ ) hat neuerdings noch über den frïher bereits von Karmán veröffentlichten Fall eine Mitteilung gemacht, hauptsächlich weil er bei dem nunmehr zehn Jahre alten Kinde die Fähigkeit zu weinen feststellen konnte. Dabei wölbt sich allmählich die die Augen bedeckende Hautbrücke vor, es fliesst dann Tränenflüssigkeit aus der Nase ab; es muss also eine subkutane Verbindung zwisclien der Tränendrüse bzw. ihren Abflusswegen und der Nase bestehen. Wie diese zu denken ist, lässt sich nicht bestimmt angeben, da bei dem frühern Operationsversuch keine Spur eines Conjunctivalsackes gefunden war.

Goldzieher schliesst sich der Auffassung an, dass der Kryptophthalmus entzündlichen Ursprungs sei, und glaubt die Tatsache, dass die bedeckende Schicht gewöhnliche Haut- und nicht Lidstruktur zeigt, in folgender Weise erklären zu können: "Nehmen wir nun an, dass um dieselbe Zeit, da der Bindehauttractus sich bildet, durch einen geschwürigen Prozess die Oberfläche des Augapfels zu Grunde geht, demnach ein grosser Substanzverlust entsteht, der zur Verklebung der Wundfläche und der sie bedeckenden Hautwülste führt, aus denen sich die Lider bilden wollen. Infolge dieser Verklebung muss die Bildung des Conjunctivaltractus unterbleiben, da eine untrennbare Narbenstrang-Verbindung zwischen Bulbus und Bedeckung zu stande gekommen ist. Mit dem Zugrundegehen ist auch das Schicksal der spezifischen Lidorgane entschieden, sie gelangen nicht zur Ausbildung......, es fehlt nun auch der embryologische Anlass zur Trennung der verklebten Lidspalte, der doch nur in der

1) Andogsky, Einige Fälle von Kryptophthalmus. St. Petersb. ophthalm. Gesellsch. 27. April 1900. Wratseh. XXI. p. 599.

2) Goldzieher, Zur Lehre von Kryptophthalmus congenitus. Centralbl.

f. Augenheilk. 1903. August. 
vollendeten Ausbildung der Lidrandgebilde zu suchen ist." Vorher sagt er noch: „Schon die teleologische Betrachtung spricht dafür, dass die Bildung der spezifischen Lidorgane, des Tarsus und der Meibomschen Drüsen mit der Bildung des Conjunctivaltractus gleichen Schritt halten muss, $d a$ diese Gebilde nur dann einen Sinn haben können, wenn eine Bindehaut existiert."1) Eine solche Betrachtungsweise ist aber nach meiner Meinung nicht berechtigt. Was haben z. B. die Augenmuskeln für einen "Sinn" bei Anophthalmus congenitus?

Zwei Fälle, die von Ginestous ${ }^{2}$ ) unter dem Namen „Cryptophthalmie congénitale" veröffentlicht sind, verdienen diese Bezeichnung nicht, da es sich um einfachen Anophthalmus eongenitus handelt.

Eine neue anatomische Untersuchung wurde in dem ersten Falle von Golowin ausgeführt:

Die bedeckende Haut ist annähernd normal, eine Narbe, welche die Spur einer Verschmelzung der Lider darstellen könnte, fehlt. Unter der Haut ein starker Muskel (Orbicularis). Knorpel, Drüsen, Conjunctiva, Tränenorgane, Levator fehlen.

An Stelle der Hornhaut findet sich Narbengewebe, das mit der bedeckenden Haut in fester Verbindung steht; im übrigen entsprechen die Veränderungen einem hochgradig phthisischen Bulbus mit Amotio retinae und von der Chorioidea ausgehender hochgradiger Verknöeherung. An den intra- sowie extraokularen Gefässen bestand ausgesprochene Endo- und Perivasculitis.

,Man kann mit grosser Wahrscheinlichkeit annehmen, dass der Prozess der Ulceration und Perforation der Hornhaut zu der Zeit begann, wo die Lider noch geschlossen waren ${ }^{3}$ ). Die nachfolgende Vernarbung rief eine vollständige Obliteration der Conjunctivalhöhle hervor und die Verwachsung der Lider mit dem Auge, dessen weitere Entwicklung gestört war." In den Gefässveränderungen will er ein Anzeichen für ein konstitutionelles Leiden sehen, das die Ursache der fötalen Augenkrankheit war.

Die Zeit der Entstehung der Anomalie verlegt Golowin zwischen den dritten und sechsten Monat, wo die Lidspalte geschlossen ist; bei früherem Eintritt der supponierten Hornhauterkrankung soll die Verwachsung eventuell nur mit einem Lid zu stande kommen.

Es besteht also nach der voranstehenden Zusammenstellung zwischen allen neuern Autoren eine - man möchte sagen erfreuliche - Übereinstimmung in der Annahme, dass der Kryptophthalmus entzündlichen Ursprungs ist. Da ich selber diese Meinung auch

1) Von mir gesperrt.

2) Ginestons, Deux cas de Cryptophthalmie congénitale. Mém. et Bull. de la soc. de méd. et de Chir. d. Bordeaux. 22. Avril 1904. Gaz. hebdom. de Bordeaux. 12. juin 1904.

3) Von mir gesperrt. 
vertreten habe, so bedaure ich es ganz besonders, jetzt nach nochmaligem eingehendem Studium der Literatur und reiflicher Erwägung besonders auch des mitgeteilten eigenen Falles jene Auffassung bekämpfen zu müssen.

Es ist zunächst ohne weiteres zuzugeben, dass eine eitrige $\mathrm{Ke}$ ratoconjunctivitis, welche $\mathrm{zu}$ sehr ausgedehnter Geschwürsbildung führt, eine mehr oder weniger vollständige Verwachsung der Lider untereinander oder gleichzeitig mit der Bulbusoberfläche herbeiführen kann. Das lehren die Fälle von sog. Kryptophthalmus cicatriciosus und ferner der von $\mathrm{Bach}^{1}$ ) beschriebene Kryptophthalmus beim Kaninchen. Dieser letztere, den ich seinerzeit zum Beweis der entzündlichen Entstehung dieser Missbildung als besonders wichtig mit herangezogen habe, muss, wie ich jetzt glaube vertreten zu dürfen, ausgeschaltet werden, da es mir sehr wabrscheinlich ist, dass es sich dort überhaupt um keine angeborene Missbildung handelt.

Der Fall betrifft ein ungefähr einjähriges Kaninchen. „Die Lidspalte war rechts vollkommen verwachsen, links fand sich nur temporal eine $21 / 2 \mathrm{~mm}$ lange Spalte .... Die Cilien waren beiderseits, jedoch nur sehr spärlich vorhanden." Anatomisch fanden sich bier Verwachsungen mannigfacher Art zwischen Ober- und Unterlid, dagegen nicht zwischen Lidern und Hornhaut, der Conjunctivalsack war normal entwickelt, die Hornhaut zeigte Residuen schwerer Entzïndung.

Klinisch unterscheidet sich dieser Fall ron allen andern dadurch, dass man den Eindruck einer verwachsenen Lidspalte hatte und dass Cilien vorhanden waren; anatomisch durch das Vorhandensein sämtlicher normaler Gebilde des obern Lides und eines geräumigen Conjunctivalsackes. Das Fehlen ron Knorpel und Drüsen am Unterlid ist allerdings auffallend. Sehen wir aber von diesem einen Punkt ab, so kann das ganze Krankheitsbild ohne weiteres durch einen Entzündungsprozess im extrauterinen Leben erklärt werden.

Ich habe vor kurzem bei drei 14 Tage alten Kaninchen eine spontan entstandene schwere eitrige Keratoconjunctivitis gesehen, welche nur eine minimale Öffnung der vor kurzem frei gewordenen Lidspalte gestattete. Im Alter von drei Wochen wurden die Tiere getötet. Die anatomische Untersuchung ergibt zwar keine Verwachsung der Lidspalte, aber noch sehr hochgradige eitrige Keratitis mit

1) Bach, Anatom. Befund eines doppelseit. angeb. Kryptophthalmus beim Kaninchen. Arch. f. Augenheilk. Bd. XXXII. S. 16. 
ausgedehnter Geschwürsbildung und Conjunctivitis mit stellenweiser Verklebung zwischen Conjunctiva tarsi und Hornhaut. Dass ein solcher Prozess unter Umständen zu einem Bilde, wie es im Bachschen Falle vorhanden war, führen kann, erscheint mir zweifellos.

Mag aber dort der zur Verwachsung der Lidspalte führende Prozess tatsächlich nach oder, wie bisher von allen angenommen ist, vor der Geburt gespielt haben, soviel ist sicher, dass er im letztern Fall erst in einem späten Stadium der Entwicklung, längst nach vollständiger Ausbildung der Lider eingesetzt hat.

Nehmen wir bei ihm die intrauterine Entstehung an, so haben wir gleich die grosse Schwierigkeit, dass wir ein Eindringen von eitererregenden Mikroorganismen in den vollkommen geschlossenen Conjunctivalsack voraussetzen müssen. Diesen Punkt, der natürlich auch für die andern Fälle gilt, möchte ich vorweg betonen.

Ganz im Gegensatz zu dem Bachschen Falle spricht in sämtlichen andern nichts dafür, dass" die Haut, welche die Angengegend überzieht, wirklich den verwachsenen Lidern entspricht; ferner ist das vollständige Fehlen eines Conjunctivalsackes entweder sicher festgestellt oder mindestens wahrscheinlich ${ }^{1}$ ). (Ich möchte Blessigs gegenteilige Annahme für seinen Fall schon deshalb für irrtümlich halten, weil es einfach undenkbar ist - welche Genese man auch annehmen mag -, dass sich beim vollständigen Kryptophthalmus eine klare Hornhaut findet, wie Blessig gesehen zu haben glaubte.) Endlich zeigten die Bulbi, soweit sie untersucht wurden, ganz andere Verhältnisse wie in Bachs Fall, wo sie im wesentlichen normal waren. Dieser teilt also mit den andern eigentlich nur die oberflächliche Ähnlichkeit, dass keine offene Lidspalte vorhanden war.

Ehe ich näher begründe, warum ich die Entstehung des typischen Kryptophthalmus glaube in eine sehr frühe embryonale Periode verlegen $z u$ müssen, möchte ich noch untersuchen, ob die erhobenen anatomischen Befunde wirklich geeignet sind, der Entzïndungstheorie als sicheres Beweismaterial zu dienen.

Eine eigentliche Narbenbildung ist an der einer "Raphe" ähnlichen Hautstelle niemals konstatiert worden; ihr Vorhandensein beweist demnach nicht, dass es sich um verwachsene Lider handelte.

1) Rein klinische Beobachtungen bei operativen Versuchen sind wegen der Schwierigkeit, die topographischen Verhältnisse richtig zu beurteilen, nicht verwertbar. 
Die Anordnung der Muskulatur kann das Auftreten einer solchen Linie, besonders beim Schreien, erklären. Dass ein Orbicularis und ein Levator vorhanden ist, braucht nicht die Ausbildung von Lidern zu beweisen (vgl. die Anwesenheit der Augenmuskeln beim Anophthalmus).

Die pathologischen Befunde an den Augen selbst sind nicht leicht sicher zu deuten; zum Teil sind auch die histologischen Untersuchungen unbefriedigend. Dass sich an Stelle der Hornhaut eine bindegewebige vaskularisierte Membran findet, braucht nicht auf Entzündung zu beruhen (siehe weiter unten), der Mangel der Iris, das Fehlen oder die rudimentäre Beschaffenheit der Linse, das Auftreten von Fett im Glaskörperraum spricht für eine Bildungsanomalie, es sind aber anderseits auch Befunde am Corpus ciliare und der Chorioidea sowie der Netzhaut beschrieben, die mit grösster Wahrscheinlichkeit oder Sicherheit als entzündliche aufgefasst werden müssen.

So wenig aber zweifellose entzündliche Veränderungen, die sich in einem mit Colobom, Mikrophthalmus oder Aniridie behafteten Auge vorfinden, den entzündlichen Ursprung dieser Missbildungen beweisen, sondern, wie wir jetzt sicher wissen, sekundär sind, so wenig beweisen Entzündungsprodukte in den degenerierten Bulbis den entzündlichen Ursprung des Kryptophthalmus, namentlich wenn wir bedenken, dass die Augen zum Teil erst in vorgerücktem Lebensalter ihrer Träger zur Untersuchung kamen; z. B. Golowins Fall I.

Es fragt sich nun weiter: ist eine Theorie, welche annimmt, dass eine an der Oberfläche des Bulbus lokalisierte Entzündung zur Verwachsung von Auge und Lidern führt, überhaupt mit den tatsächlichen Befunden beim Kryptophthalmus vereinbar?

Wir scheiden hier zwei Gruppen: Eintritt der Entziindung 1. nach bereits erfolgtem Schluss der Lidspalte, 2. vor demselbẹn.

Bei der unter 1. gemachten Voraussetzung ist es absolut ausgeschlossen, dass eine totale Verwachsung des obern Lides mit der Bulbusoberfläche bei vollkommen normaler Beschaffenheit des untern samt seinem Conjunctivalsack resultieren kann. Wenn wir aber diesen Befund auf der einen, einen vollständigen Kryptophthalmus auf der andern Seite haben, so müssen wir die gleiche Erklärung für beide Seiten anwenden können. Es ist ferner bei jener Voraussetzung völlig unmöglich, dass ein Bild entsteht, wie es sich auf der linken Seite in meinem Falle findet: normale Ausbildung eines temporalen

v. Graefe's Archiv für Ophthalmologie. LXחI. 1. 
Stückchens vom obern und untern Lide mit dazwischenliegendem Conjunctivalsack, während die ganze übrige Lidspaltengegend von gleichmässiger Hautschicht bedeckt ist. Wie sollte eine destruierende Entzündung, die sich innerhalb des geschlossenen Conjunctivalsackes abspielt, derartige Bilder: absolute Verödung des Conjunctivalsackes unmittelbar angrenzend an vollkommen normale Teile, herbeiführen können?

Wir müssen also, wenn wir alle Fälle von Kryptophthalmus einheitlich auffassen wollen, eine Entstehung nach Schluss der Lidspalte für ausgeschlossen halten.

Bleibt also die Möglichkeit, dass eine oberflächliche Entzündung zu einer Verwachsung mit den bereits gebildeten, aber noch nicht zur Verwachsung gelangten Lidern führt. In diesem Falle ist ein vollständiger Kryptophthalmus kaum erklärbar, ferner schon sehr schwer die Verwachsung des obern Lides mit der Bulbusoberfläche bei normaler Ausbildung des untern Lides. Denn man muss sich doch klar machen, dass bei den überaus kleinen Verhältnissen, welche die Teile in diesem Entwicklungsstadium noch zeigen, ein derartig umschriebener Eiterungsprozess ohne Schädigung der Nachbarschaft, wie man ihn hier voraussetzen müsste, kaum möglich ist.

Vollends unmöglich erscheint es mir aber, auf diese Weise zu einer Erklärung meines oben genannten Befundes: - normale Beschaffenheit des temporalsten Teils beider Lider bei völliger Überhäutung des übrigen Lidspaltenbezirkes - zu gelangen.

Für diese Beobachtung versagt also meines Erachtens die Entzündungstheorie absolut, und da wir für sie keine spezielle Erklärung aufstellen dürfen, so kommen wir dahin, die Entstehung des Kryptophthalmus in die Zeit vor der Bildung der Lider zu verlegen; in diesem Stadium ist die Annahme einer eitrigen Entzündung, die zur Bildung des Kryptophthalmus führen könnte, schon von vornherein ausgeschlossen, ebenso wie sie nicht in Betracht kommt für die Syndactylie und die Missbildung der Genitalien, beides Dinge, die sich so oft mit Kryptophthalmus gemeinsam finden, dass ein zufälliges Zusammentreffen kaum annehmbar erscheint ${ }^{1}$ ). Ich kann den Kryptophthalmus nur dadurch erklären, dass die Bildung der Lider ganz oder teilweise auf mechanische Weise verhindert worden ist und spreche mit diesem Satze ja nur eine Vorstellung aus, die schon einmal Bürgerrecht besass, dann aber

1) Ich setze mich mit diesen Ausführungen in Gegensatz zu frühern. 
durch die Entzündungstheorie verdrängt wurde. Ein Ausbleiben der Lidentwicklung durch "innere Ursachen" kann ich nicht annehmen, weil dadurch niemals mein mehrfach citierter Befund erklärt werden könnte. Wir müssen deshalb nach einem Faktor suchen, welcher im stande ist, auf mechanischem Wege die Ausbildung der Lider ganz oder teilweise zu verhindern. Von solchen Faktoren können wir, soweit ich wenigstens orientiert bin, zurzeit nur Anomalien des Amnion heranziehen und ich komme damit wieder auf v. Duyses ältere Anschauung, die später noch von $K u n d r a t$ vertreten wurde, zurück. Wenn die angenommene Wachstumsbehinderung vol Bildung der Lider eintritt, so muss dies spätestens am Anfang bzw. in der Mitte des zweiten Monats geschehen, da die Entwicklung der Lider beim Menschen nach v. Ammon im zweiten Monat beginnt, nach Kölliker bei einem 8-9 Wochen alten Embryo schon recht weit vorgeschritten war. Es erscheint denkbar, dass ein solches Hindernis für die ganze Augengegend vorhanden war, ebenso aber, dass es etwa in Gestalt einer vertikal verlaufenden Amnionfalte einen Teil der Augengegend unbehelligt liess, so dass sich wie in meinem Falle der am weitesten temporal gelegene Teil beider Lider bilden konnte. Ebenso kann man in dieser Weise eine normale Entwicklung des untern Lides verstehen, während an Stelle des obern ein mit dem Bulbus in festem Zusammenhang stehendes Hautstïck gefunden wird. Nach dieser Auffassung handelt es sich dann auch gar nicht um eine Verwachsung zwischen Bulbus und Lidern bzw. einer an ihrer Stelle befindlichen Hautpartie, sondern um einen abnormen Entwicklungsvorgang, der es bedingte, dass Bulbus und überziehende Haut von vornherein in direkter Verbindung miteinander geblieben sind. Diese Entwicklungsstörung kann sowohl nach wie vor der Zeit der Linseneinstülpung erfolgen und letztere eventuell unterbleiben. Aus dem die Augenanlage vorn bedeckenden Mesoderm und Ektoderm wird sich nicht wie normal Cornea und ihr Epithel, sondern gerade wie in der Umgebung Haut mit ihren verschiedenen Bestandteilen differenzieren, höchstens werden sich die tiefsten Lagen des Mesoderms zu Cornealgewebe umbilden, auf keinen Fall kann aber eine vorn wirklich normal abgegrenzte klare Hornhaut resultieren; das stimmt auch mit den Befunden überein, wenn man den Bachschen Fall in der Weise, wie ich es getan habe, absondert und zeigt zugleich, dass operative Versuche zur Wiederherstellung von Sehvermögen in Fällen von echtem Kryptophthalmus zwecklos sind, höchstens können sie zur Kontrolle der entwickelten Anschau- 
ungen auf ihre Richtigkeit dienen. Differenzieren sich die tiefsten Mesodermschichten wirklich zu Cornealgewebe, so ist die Bildung einer vordern Kammer denkbar und der Ausbildung der übrigen Teile steht a priori kein schweres Hindernis entgegen, abgesehen vielleicht von dem Druck, den das Amnion ausübt. Es ist aber auch sehr wohl möglich, dass eine eigentliche Cornea und vordere Kammer überhaupt nicht entstehen und dass die das Auge deckende Hautlage nur durch den Inhalt des Glaskörperraums vorgewölbt wird und auf diese Weise ein Hohlraum entsteht, den man irrtümlich für eine vordere Kammer halten könnte. Dass ein solches völliges Ausbleiben der Bildung einer Cornea vorkonmt, werde ich im folgenden Abschnitt an der Hand eines Falles, der wohl mit dem Kryptophthalmus in naher Beziehung steht, näher zeigen können.

Es mag nicht unerwähnt bleiben, dass die Syndactylie und die Missbildungen an den Geschlechtsteilen auch mit hoher Wahrscheinlichkeit auf Anomalien des Amnions znrückgeführt werden und dass wir so eine einheitliche Erklärung gewinnen köınen, die ich früher für unmöglich hielt.

Nachträglich finde ich, dass in einer Leipziger Dissertation von Schiff ${ }^{1}$ ) in einem Falle von sogenanntem Symblepharon congenitum des obern Lides wegen Vorhandensein noch anderer Spuren amniotischer Verwachsungen die Anschauung geäussert worden ist, dass sowohl jener Fall wie der Kryptophthalmus auf die Einwirkung des Amnion zu beziehen sei. Die prinzipielle Verschiedenheit der dort geäusserten Auffassung gegenüber der meinigen liegt aber darin, dass der Autor eine Verwachsung des Bulbus mit den zur Ausbildung gelangten Lidern annimmt, die er in den dritten bis vierten Monat verlegt, während ich aus den angeführten Gründen der Ansicht bin, dass die letztere überhaupt nicht erfolgt ist.

Eine besondere Berücksichtigung erfordert an dieser Stelle noch die interessante Arbeit von Matys ${ }^{2}$ ), welcher bei einem viermonatlichen menschlichen Embryo einen amniotischen Strang beschreibt. „der in der rechten Nasengegend beginnend, das Unterlid an dieselbe, zugleich aber teilweise auch das Unterlid an die Sklera und Cornea befestigt, sodann weiter fortschreitet, indem er das ganze Oberlid in

1) Schiff, Über einen Fall von Symbleph. congen. des obern Lides, Syndactylie und Hypospadie. Inaug.-Dissert. Leipzig 1902.

2) Matys, Eine Missbildung des Auges, bedingt durch ein amniotisches Band. Zeitschr. f. Augenheill. Bd. XIII. 2. S. 150. 
sich aufnimmt und gegen die Stirne hinaufzieht, auch Teile der Supercilien erfasst, und nachdem er die Stirngegend erreicht" hatte, frei wird und dem Amnion zustrebt".

Das Auge selbst war, abgesehen von der teilweisen Verbindung mit dem untern Lid, vollkommen normal entwickelt, die Lider waren durch die normale Epithelleiste verklebt und der geräumige Conjunctivalsack durch den Zug des Stranges erheblich in distaler und medialer Richtung ausgezogen. Auch die Teile des Auges selber waren etwas in der Zugrichtung des Stranges verlagert.

Die Fixierung des Stranges verlegt der Autor in die Zeit vor der Bildung der Lider und nimmt an, dass eine Amnionfalte an der Stelle, wo sich der Oberkieferfortsatz des ersten Kiemenbogens mit dem Stirnfortsatz berührt, ganz oberflächlich aufgenommen sei.

„Das so befestigte Amnionband drückte auf jenen Teil des Gesichtes, wo sich das untere Angenlid entwickelt und zwar auf den medialen Anteil dieses Lides. Das Lid selbst entwickelte sich weiter, wuchs vor den Bulbus, und da es durch den stetigen Druck des Stranges an den Bulbus angedrïckt wurde, verwuchs es mit dem letztern im Bereich des Stranges, ursprünglich nur mit der Sklera, später auch mit der Cornea. Durch das Wachstum des Kopfes wurde nunmehr auch das obere Augenlid gegen jenen Strang gedrückt, der schliesslich mit demselben in seiner ganzen Breite verwuchs. . .."

Gegen die Annahme, dass die Fixation des Stranges erst nach Ausbildung der Lider erfolgt sei, spricht nach Matys die Tatsache, dass an der Verwachsungsstelle des Unterlides mit Sklera bzw. Cornea keine Epithelzelle und keine Gewebsverdickung vorhanden war. Auch auf dem Lide selber hätte man den Strang bei späterer Verwachsung wenigstens als besondere Hervorragung erkennen müssen, was nicht der Fall war.

Ich weiss nicht, ob diese Argumente entscheidend sind; am obern Lid muss doch auch nach des Autors eigener Annahme eine nachträgliche Verwachsung zweier mit Epithel bekleideter Flächen ohne Zurückbleiben von Epithelzellen angenommen werden, also hätte eine solche wohl auch zwischen Unterlid und Bulbus erfolgen können. Für die Annahme einer Verwachsung nach Ausbildung der Lider könnte man wohl die normale Ausbildung des Auges und der Lider mit Conjunctivalsack (ausschliesslich der Verwachsungsstelle) anführen.

Doch liegt es mir völlig fern, die Richtigkeit von Matys' Erklärungsart in Abrede stellen zu wollen. Trifft sie zu, so war der Strang nach der Art seiner Anheftung nicht geeignet, die Ausbildung 
der Lider zu verhindern oder die Entwicklung des Auges erheblich zu beeinträchtigen. Es ist demzufolge auch ein vom Kryptophthalmus sehr verschiedener Befund zu stande gekommen. Dennoch ist der Fall auch für diese Missbildung von Interesse, weil er aufs neue das tatsächliche Vorkommen amniotischer Verwachsungen in der Gegend des Auges beweist. Denkt man sich die Ausdehnung und Befestigungsweise des Stranges in etwas anderer Art, als Matys sie gefunden hat, so ist die Möglichkeit der Entstehung des Kryptophthalmus ohne weiteres einzusehen.

Es soll schliesslich noch darauf hingewiesen werden, dass der Druck des Amnion eventuell erst nach Ausbildung der Lider und des Conjunctivalsacks zu Kryptophthalmus führen könnte. Dies wäre dann denkbar, wenn man annimmt, dass er zu völliger Zerstörung des conjunctivalen und cornealen Epithels und folgender Verwachsung der mesodermalen Bestandteile des Lides mit dem Bulbus führt. Dabei wäre auch die Möglichkeit gegeben, dass ein Teil der Lider von diesem Druck nicht getroffen wird, so dass ein Bild wie in meinem Falle am linken Auge entstehen könnte. Ich halte aber eine solche Auffassung, die der von Schiff geäusserten entspricht, mit Rücksicht auf die obigen Ausführungen für wenig wahrscheinlich; ausschliessen lässt sie sich vor der Hand nicht.

\section{Epibulbäres Dermoid, Lidcolobom und Mikrophthalmus.}

(Hierzu Fig. 10 und 11 anf Tafel II u. III.)

Im August d. J. hatte ich Gelegenheit, folgenden Fall zu operieren :

E. G., ein Jahr alt. Eltern gesund, ebenso das ältere Kind derselben. Im dritten Monat der Schwangersehaft fiel die Mutter des Patienten beim Treppensteigen, ohne sich aber weiter zu beschädigen. Nachher Schwangerschaft normal. Gleich nach der Geburt bemerkten die Eltern die jetzt nachweisbare Gesehwulst in der linken Lidspalte; anfänglich sei die letztere geschlossen gewesen, habe sich aber nach etwa drei Wochen geöffnet, die Geschwulst sei um diese Zeit etwas gewachsen. Seit der Geburt soll am untern Lidrand etwas gelbliche Flüssigkeit abgesondert sein.

Status praesens. Gesund aussehendes Kind. Rechtes Auge normal. Links: Die Lidspalte ist ausgefüllt von einer rötlichgelben Geschwulst, deren Oberfläche zum Teil deutlichen Epidermischarakter (Härehen) zeigt. Bei Bewregungen des rechten Auges sieht man die Geschwulst sich mitbewegen. Das obere Lid zeigt einen Defekt von $1 \mathrm{~cm}$ Länge, an dieser Stelle fehlt der Tarsus vollkommen, ebenso die Cilien; daselbst geht die Haut direkt in die Conjunctiva über, während nach aussen davon ein 
Stück normalen Oberlides von $1 \mathrm{~cm}$ Länge, nasalwärts nur ein ganz kleiner Stumpf eines Lides sich anschliesst. Ein Canthus externus fehlt, die Conjunctiva bulbi geht hier direkt in die äussere Haut über, der Befund hat Ähnlichkeit mit dem Bilde, das nach einer sehr ausgiebigen Canthoplastik entsteht. Vom temporalen Ende des untern Lides geht eine Hautfalte auf den Tumor über.

Der obere Tränenpunkt ist nicht mit Sicherheit $\mathrm{zu}$ erkennen, am untern Lid findet sich dagegen $1 \mathrm{~cm}$ vom Canthus internus entfernt ein im intermarginalen Teil gelegener Tränenpunkt, aus dem sich Eiter entleert. In Narkose kommt man mit feiner Sonde nicht in den Tränensack, sondern hat den Eindruck, dass das Tränenröhrchen blind endigt. Die Entleerung von Eiter spricht aber dafür, dass eine Schleimhautfalte den Tränensack verschliesst. Unmittelbar nach aussen vom linken Nasenflügel findet sich eine trichterförmige Einziehung der Haut, die blind zu endigen scheint.

Am untern Rand des linken Nasenlochs auf das Septum übergreifend findet sich eine häutige, erbsengrosse Verdickung, an dieser Stelle soll einige Tage nach der Geburt ein gestielter Anhang abgetragen sein.

Diagnose: Epibulbäres Dermoid auf rudimentärem Bulbus, Colobom des obern Lides und der äussern Kommissur, temporale Verziehung des untern Tränenröhrchens, Reste amniotischer Verwachsungen an Nase und Wange.

Aus kosmetischen Griinden wird die Enucleation ausgeführt, dabei wird ein deutlicher, weiss aussehender Sehnerv durchtrennt. Durch Präparation kann man sich leicht davon überzeugen, dass ein kleiner Bulbus mit deutlicher Sklera vorhanden ist.

Härtung in Formol, Celloidin. Serienschnitte parallel zum vertikalen Meridian.

Der kleine Bulbus hat länglich ovale Form. Misst man in den Schnitten, welche den Sehnerven enthalten, den einwärts von der Sklera gelegenen Bulbusinhalt, so beträgt seine Länge $5 \frac{1}{2}$, seine Höhe $3 \frac{1}{2} \mathrm{bm}$; der hintere Teil des Bulbus bis zum Corpus ciliare hin ist von einer $1 \mathrm{~mm}$ dicken Sklera eingeschlossen. Der regelmässig kreisrunde Sehnerv hat einen Durchmesser von $11 \% \mathrm{~mm}$ ausschliesslich der Scheiden. Makroskopisch ist an dem Bulbus eine Chorioidea, Corpus eiliare, Pigmentepithel zu erkennen, fast der ganze Innenraum scheint von der faitig zusammengelegten Retina eingenommen $\mathrm{zu}$ sein, von einer linse ist nichts $\mathrm{zu}$ sehen, ebensowenig von vorderer Kammer oder Cornea. Sehr bemerkenswert ist die Form des Bulbus: dieselbe erinnert ausserordentlich an das Verhalten der sekundären Augenblase, d. h. oben reicht die Bulbuswand viel weiter nach vorn als unten, wo das Corpus ciliare erheblich zurücktritt, das Gewebe des Der. moids erstreckt sich demzufolge unten erheblich weiter nach hinten als oben. Nach vorn sitzt dem Bulbus ein im anteroposterioren Durchmesser ca. 11, von oben nach unten ca. $15 \mathrm{~mm}$ messendes Dermoid auf, dessen grösster Teil aus Fettläppchen, die von Bindegewebszügen durehzogen sind, 
besteht, während die Oberfäche schon makroskopisch deutlichen Hauteharakter erkennen lässt.

Mikroskopischer Befund: Wegen ihrer Wichtigkeit soll die Schilderung der topographischen Verhältnisse in den Vordergrund gestellt werden. Obgleich nach meinen frühern Darlegungen die sagittale Schnittführung zur Erkennung kleiner Colobome nicht günstig ist, lässt sich doch, weil ich jeden zweiten bis dritten Schnitt eingelegt habe, an drei Präparaten mit Sicherheit erkennen, dass unten ein kleines Colobom des Corpus eiliare besteht. Durch diesen Spalt zieht ein bindegewebiger Strang von unten her in das Innere des Bulbus und verästelt sich hier in ganz ähnlicher Weise, wie ich dies in meinen früher beschriebenen Mikrophthalmen gefunden habe; er steht ferner in direkter Verbindung mit einer bindegewebigen Masse, welche die vordere Öffnung des Augenbechers ausfüllt.

Diese Öfnung ist nur in den Sehnitten aus der Mitte des Bulbus vorhanden und wird eingefasst von dem Corpus eiliare. Von einer Vorderkammer oder Hornhaut ist keine Spur nachweisbar, sondern nach vorn grenzt direkt an die wenig entwickelte Iris bzw. das Corpus ciliare das lockere, von Fettläppchen durchsetzte Bindegewebe der tiefsten Schichten des Dermoids, und innerhalb derselben befindet sich ein ganz deutliches Linsenrudiment. Dasselbe liegt also vor dem Bulbus und ist nicht in denselben eingetreten. Es besteht aus einer sehr deutlichen, mässig stark gefalteten Linsenkapsel mit regelmässigem Epithelbelag, der sie ringsherum überzieht, der Inhalt ist vollständig homogen und lässt nur an einzelnen Stellen unmittelbar auf dem Epithel ein Häufchen von Zellen erkenmen, die kleiner sind als die Epithelzellen, ferner sieht man einzelne runde tropfenartige Gebilde darin, von Linsenfasern dagegen ist keine Spur zu erkennen.

Die Sklera zeigt im obern Umfang normales Verhalten, unten besteht sie aus kernarmem Bindegewebe von beträchtlicher Dicke, das durch ganz unregelmässige Richtung seiner Bündel und ferner dadurch ansgezeichnet ist, dass eine scharfe Begrenzung nach aussen fehlt, vielmehr hier die derbern Bindegewebsbalken des Dermoids direkt aus dem Skleralgewebe hervorgehen.

Die Chorioidea ist breit, die Blutgefässe sehr stark gefüllt, die Stromazellen gut pigmentiert, kurz sie macht einen durchaus normalen Eindruck.

Das Corpus eiliare ist ebenfalls sehr gut entwickelt, die Muskelbündel haben eine sehr mannigfaltige Verlaufsweise, so dass man Längs-, Schrägund Quersehnitte von Kernen durcheinander antrifft. Die Ciliarfortsätze sind gut, stellenweise sogar auffallend stark entwickelt.

In den Schnitten aus der temporalen Hälfte des Bulbus ist eine Iris nachweisbar, ihre Pigmentschicht ist etwas unregelmässig, das Stroma ist im unmittelbaren Anschluss an das Corpus ciliare ziemlich breit, weiter nach vorn aber nur ganz schmal. Der Verlaut der Iris ist nicht gestreckt, sondern zeigt oben sowie unten unmittelbar vor dem Corpus ciliare eine starke Aussackung mit nach vorn gerichteter Konvexität; in den Schnitten aus der Mitte des Bulbus ist gar keine Iris vorhanden, das Corpus ciliare reicht hier weiter nach vorn und bildet den Rand der Öffnung, dureh welche 
das intraokulare Bindegewebe mit dem Gewebe des Dermoids in Verbindung steht.

Der ganze Binnenraum des Auges wird von der in massenhaften Falten gelagerten Retina eingenommen, an Stelle des Glaskörpers, ron dem nur Spuren nachweisbar sind, findet man die oben beschriebenen verzweigten Bindegewebszüge, die mit der Retina in Verbindung stehen.

An der Öfnung des Augenbechers ist der Rand der Augenblase Übergangsstelle von Pigmentepithel in Retina - stark ins Innere des Auges umgebogen. Das Pigmentepithel geht hier in hohe Cylinderzellen vom Charakter der Pars eiliaris über. Letztere bilden stark gewundene Bänder, die auf dem Durchschnitt in verschiedensten Figuren erscheinen. Die Retina selber lässt besonders da, wo sie dem Pigmentepithel anliegt, aber auch an vielen andern Stellen, die sämtlichen im wesentlichen normalen Schichten erkennen. Selbstverständlich ist eine genaue Beurteilung ibrer Bauart dadurch unmöglich, dass man so gut wie nirgends exakte senkrechte Sehnitte durch die Membran vor sich hat, immerhin ist es interessant, dass man z. B. an einer Stelle eine solche Anhäufung von Ganglienzellen nachweisen kann, dass man versucht ist, dies für die Maculagegend zu halten. Ferner ist das deutliche Vorbandensein der Nervenfaserschicht von Wichtigkeit. Zahlreich sind die sog. Rosetten.

Der Sehnerv ragt ziemlich weit ins Innere des Bulbus, wie ich es auch sonst bei meinen Untersuchungen über Mikrophthalmus gefunden habe, die Faserbündel in demselben sind ziemlich schmal, die Kernreihen sehr dicht, aber die sämtlichen Faserbündel geben tadellose Markscheidenfärbung: nach Weigert. Der Querschnitt des Nerven ist kleiner als der eines normalen gleichen Alters, aber die Struktur desselben ist der Hauptsache nach normal.

Die Pigmentepithelschicht der Retina ist überall vorbanden und zeigt stellenweise kleinere und grössere Excrescenzen. Erhebliche Veränderungen fehlen an derselben.

Das Dermoid zeigt ein sehr mächtiges subcutanes Fettpolster, die Hautschicht weist eine dicke Epidermis, Papillen, Talg- und Schweissdrüsen, sowie Haare auf.

Endlich sei noch bemerkt, dass ich an zahlreichen arteriellen Gefässen, die zu den Seiten des Sehnerven verlaufen, ausgesprochene Endarteriitis obliterans gefunden habe. Dieser Befund stimmt überein mit dem bei einem andern Mikrophthalmus ${ }^{1}$ ) erhobenen sowie mit dem Golowins ${ }^{2}$ ) bei seinem Kryptophthalmus. Letzterer Autor schliesst daraus auf das Vorhandensein einer konstitutionellen Krankheit. Ich möchte mich begnügen, den Befund vorläufig nur zu registrieren.

\section{Epikrise.}

Auf der linken Seite finden wir: einen grossen Defekt am obern Lid, Fehlen der äussern Lidkommissur, Verziehung, des untern Tränen-

1) E. v. Hippel, Ist das Zuisammenvorkommen von Mikrophthalmus congenitus und Glioma retinae im gleichen Auge sicher erwiesen? v. Graefe's Arch. f. Ophthalm. Bd. LXI. 2. 2) Loc. cit. 
röhrchens temporalwärts, Residuen amniotischer Verwachsungen an Nase und Wange, grosses epibulbäres Dermoid, das einem ausgesprochenen Mikrophthalmus aufsitzt. Der letztere zeigt ein kleines Ciliarkörpercolobom, einen Bindegewebsstrang, der durch dasselbe in den Bulbus eintritt, sich dort verzwejgt und ausserdem mit dem Dermoid zusammenhängt, fast vollständiges Fehlen des Glaskörpers, hochgradige Faltenbildung der wenig veränderten Retina, nahezu normale Beschaffenheit des Optikus, der Chorioidea und des Corpus ciliare. Die Linse liegt vor der Öffnung des Augenbechers innerhalb der tiefsten Schichten des Dermoids.

Betrachten wir zunächst den Mikrophthalmus: wir können ihn genau so auffassen, wie ich es in meinen frühern Arbeiten getan habe. Das kleine Ciliarkörpercolobom zeigt die Störung im Schluss der Fötalspalte an; die fehlende Bildung des Glaskörpers bedingt, dass die Augenblase, der wir eine, wenn auch nicht normale, so doch eine sehr erhebliche Wachstumstendenz zuschreiben können, keinen Platz findet, sich glatt auszubreiten, daher die massenhaften, ins Innere des Bulbus steigenden Falten, die also keinesfalls als der Ausdruck einer sekundären pathologischen "Netzhautablösung“ angesehen werden dürfen. Die auffallend gute Erhaltung der histologischen Struktur der Membran passt sehr gut zum nahezu normalen Verhalten des Sehnerven. Dass das Mesoderm hier nicht nur durch den Spalt im Ciliarkörper, sondern auch in breitem Zuge durch die vordere Öffnung des Augenbechers ins Innere des Bulbus tritt, berubt darauf, dass der normalerweise bestehende, nahezu vollständige Abschluss der letztern durch die Linse in unserem Falle fehlt.

Wir erhalten durch unsern Befund eine neue Bestätigung der schon bekannten Tatsache, dass eine Einstiulpung der Augenblase von vorne her auch ohne Mitwirkung der Linse zu stande kommen kann.

$\mathrm{Da}$ wir eine Störung im Schlusse der Augenspalte, ferner eine solche in der Abschnürung der Linse vor uns haben, so können wir die Zeit, in der unsere Missbildung entstanden ist, ziemlich genau angeben: das Ende des ersten Monats.

Warum die Linse nicht ins Innere des Auges gelangt ist, sondern vor demselben liegen geblieben ist, können wir nur vermuten. Am nächsten scheint mir die Annahme zu liegen, dass sich ihre Abschnürung vom Ektoderm nicht in normaler Weise vollzog, sondern dass sie mit demselben zu lange in Verbindung blieb. Inzwischen hatte sich schon reichlicheres Mesoderm zwischen Augenblase und Linse eingeschoben. Die Erklärung ist natürlich hypothetisch. Wie 
der Befund aufzufassen ist, dass man an der Linse keine Spur von Fasern, sondern nur einen von Epithel ausgekleideten Kapselsack mit homogener Inhaltsmasse findet, lasse ich dahingestellt. Soll man darin eine Erhaltung des Zustandes unmittelbar nach Abschnürung des Linsenbläschens nur unter Vergrösserung der Dimensionen und Abscheidung einer deutlichen Kapsel, oder das Ergebnis einer totalen Verflüssigung der Fasermasse erblicken?

Das Linsenrudiment liegt innerhalb des Dermoids. Da die Sklera sich nach vorne unmittelbar in das letztere fortsetzt, von einer Cornea aber auch nicht andeutungsweise die Rede ist, so müssen wir annehmen, dass das die Linse einschliessende Mesoderm mit dem Ektodermüberzug sich zu Haut differenziert hat.

Das ursächliche Moment dieses abnormen Entwicklungsganges dïrfte mit grosser Wahrscheinlichkeit in amniotischen Verwachsungen oder dem Druck amniotischer Bänder zu erblicken sein. Die Spuren derselben finden wir in dem warzenartigen Auswuchs an der Nase, der blindsackartigen Grube der Wange, der Verziehung des Tränenpunktes nach aussen, dem Fehlen der äussern Kommissur und dem grossen Defekt im obern Lide.

Diese Befunde an den Lidern sind besonders interessant, weil sie uns die nahen Beziehungen zwischen epibulbärem Dermoid, Lidcolobom, den von oben auf den Bulbus übergehenden Hautbrücken und endlich dem Kryptophthalmus zeigen.

In unserem Falle hat der angenommene amniotische Strang die Ausbildung des obern Lides nur an umschriebener Stelle verhindert, es ist zum epibulbären Dermoid gekommen; wäre das Hindernis etwas ausgedehnter, so hätten wir eine von oben herkommende, mit der Bulbusoberfläche verwachsene Hautplatte, kein oberes Lid. Bei noch grösserer Ausdehnung würde überhaupt keine Lidbildung eintreten können und wir hätten den Kryptophthalmus, wie es im vorigen Abschnitt auseinandergesetzt wurde ${ }^{1}$ ).

Wir werden es dann auch nicht wunderbar finden, wenn die Beschaffenheit des Bulbus bei diesen verschiedenen Missbildungen weitgehende Übereinstimmung zeigt, und ich glaube in der Tat, dass eine solche vorliegt. Betreffs der Cornea verweise ich auf das im

1) Ich möchte in diesem Zusammenhang noch einmal darauf hinweisen, dass bei dem schon citierten Falle von de Waele und Lewouillon ausser dem doppelten Lidcolobom der Canthus externus fehlte, dass ferner an der Nase eine Hautprominenz und auf der Wange warzige Erhebungen da waren, also fast genau der Befund wie in meinem Falle. 
vorigen Kapitel Gesagte. Ich glaube auch, dass mebrere der anatomisch untersuchten Bulbi bei Kryptophthalmus typische Mikrophthalmen und nicht phthisische Augen sind. Ich verzichte aber darauf, dies hier eingehender zu begründen. Wer sich mit diesen Dingen näher beschäftigt hat, weiss, wie schwierig die Deutung der anatomischen Befunde eines einzelnen Falles ist, besonders wenn ihn ein anderer beschrieben hat. Ein sicheres Urteil kann meines Erachtens nur ein Untersucher abgeben, der die Präparate möglichst zahlreicher Fälle selber sehen und mit den eigenen vergleichen kann, und eine solche Konzentration des seltenen Untersuchungsmaterials wäre sicher für die Sache von Nutzen. Manches, was sich bei der Untersuchung nur einer Missbildung schwer oder gar nicht deuten lässt, kann im Zusammenhang mit zahlreichen andern Präparaten klar werden.

Da in unserem Falle, wo man auf die ursächliche Bedeutung amniotischer Verwachsungen geradezu hingedrängt wird, ein Colobom vorlag, so ist es natürlich naheliegend, meine früher geäusserte Ansicht ${ }^{1}$ ), wonach eine Beziehung zwischen amniotischen Störungen und Colobom nicht besteht, aufs neue zu prüfen. Muss man sich doch darüber klar sein, dass auf dem Gebiete der Missbildungen noch so vieles Hypothese, so weniges Tatsache ist, und jeden sichern positiven Befund benutzen, um weiter zu kommen. Wenn jemand im vorliegenden Falle Colobom und amniotische Anomalien zusammenbringen will, so wird man ibn nicht widerlegen können, jch gebe aber zu bedenken, dass in meinen embryologischen Untersuchungen eine solche Bezichung nicht nachweisbar war und dass dort das ursächliche Moment mit Sicherheit in einer väterlichen Keimesanomalie lag, also eine "innere Ursache" darstellte. Wollte man also dort auf das Amnion zurückgreifen, so konnte dies nur unter der Annahme geschehen, dass die Keimesanomalie eine amniotische Störung, diese aber das Colobom herbeiführt. Dies scheint mir auch heute noch keine wahrscheinliche Annahme zu sein.

Aus der neuern Literatur ist als die weitaus wichtigste Arbeit über unsern Gegenstand die von Hanke ${ }^{2}$ ) zu nennen. Ein Vergleich seines Falles mit dem meinen ergibt eine erstaunliche Ähnlichkeit der Befunde. Hier wie dort ein mesodermales Gewebe, das durch die vordere Öffnung des Augenbechers in den Bulbus tritt, nur kommt in meinem Falle noch das kleine Colobom hinzu. In beiden Fällen

1) E. v. Hippel, Embryologische Untersuchungen über die Genese der angeborenen Spaltbildungen. v. Graefe's Arch. f. Ophthalm. Bd. LV.

2) Hanke, Dermoid der Cornea, Mikrophthalmus, Aphakia congenita, Endobulbäres Lipom. v. Graefe's Arch. f. Ophthalm. Bd. LVII. 1. S. 38. 
ähnliches Verhalten der Iris, der Retina. Bei Hanke wiesen allerdings Retina und Optikus totale Degeneration auf, es handelte sich aber um ein 14jähriges Individuum; die Veränderungen samt der chorioidealen Knochenneubildung dürften demnach sekundäre sein. Beiden Fällen gemeinsam ist die enorme Grösse des Dermoids. Interessant ist es, dass bei Hanke die Linse überhaupt nicht gebildet wurde, während sie in meinem Falle rudimentär blieb und nicht bis in den Bulbus hineingelangte. Hankes Erklärungsweise stimmt in vielen Punkten mit der meinigen überein, und ich betone deshalb ausdrücklich seine Priorität, bemerke aber, dass ich mir meine Auffassung ganz unabhängig auf Grund der Befunde beim Kryptophthalmus und bei dem Dermoid gebildet und seine Arbeit erst nachträglich genauer gelesen habe. Um so erfreulicher die Übereinstimmung. Von Störungen, die auf das Amnion zurückgeführt werden könnten, sind in Hankes Fall Hasenscharte und Wolfsrachen zu nennen.

Collombi) hat noch neuestens einen Fall beschrieben, den ich nur aus dem Referat kenne: soviel ich sehe, handelt es sich um ein Dermoid der Sklerocornealgrenze, das ich erwähne, weil ausserdem doppelseitiges Dermolipom der Bindehant, symmetrische Verbildung der Ohrmuscheln, Hautgeschwulst vor dem Tragus, linksseitiger angeborener Klumpfuss vorhanden war. Der Fall illustriert also auch die Entstehung durch Anomalien des Amnion.

Die einschlägigen Fälle aus der ältern Literatur sind von mir in Graefe-Saemisch aufgezählt.

Heidelberg, 15. November 1905.

Erklärung der Abbildungen auf Taf. I-III, Nr. 1--11.

Fig, 1 bis 8 beziehen sich auf das Teratom der Orbita.

Fig. 1. Übersichtsbild: hinterer Bulbusabschnitt und angrenzender Teil der Geschwulst mit der rudimentären Augenanlage $(a$.$) , Cysten, hyaliner Knorpel.$

Fig. 2. Angenanlage bei stärkerer Vergrösserung: $a$. Retina, $b$. Pigmentepithel.

Fig. 3. Streifen von Zentralnervensystem (wesentlich Gliagewebe).

Fig. 4. Plexus chorioidens.

Fig. 5. Gliagewebe mit Neuroepithelnestern.

Fig. 6. Hohlräume mit Epidermiszellen.

Fig. 7. Darmzottenähnliche Bildung mit hohem Cylinderepithel.

Fig. 8. Übersichtsbild: $a$. Neuroepithel. b. Drüsengewebe. $c$. Darmepithel.

Fig. 9. Schnitt aus der Encephalocele der linken Seite; piä̈hnlicher Überzug, daran anstossend nervöses Gewebe mit ganz verschiedenem Zellreichtum.

Fig. 10. Übersichtsbild des Mikrophthalmus mit dem Dermoid: a. Linsenrudiment, $b$. vordere Öffnung des Augenbechers begrenzt vom Corpus ciliare.

Fig. 11. Vorderer Teil des Bulbus mit davor gelegenem Linsenrudiment bei stärkerer Vergrösserung.

1) Collomb, Beitr. zur Kenntnis der Dermoide der Conjunctiva bulbi u. Cornea. Soc. franç. d'Ophtalm. 3. Mai 1905. Refer. in Ophth. Klinik 1905. S.265. 

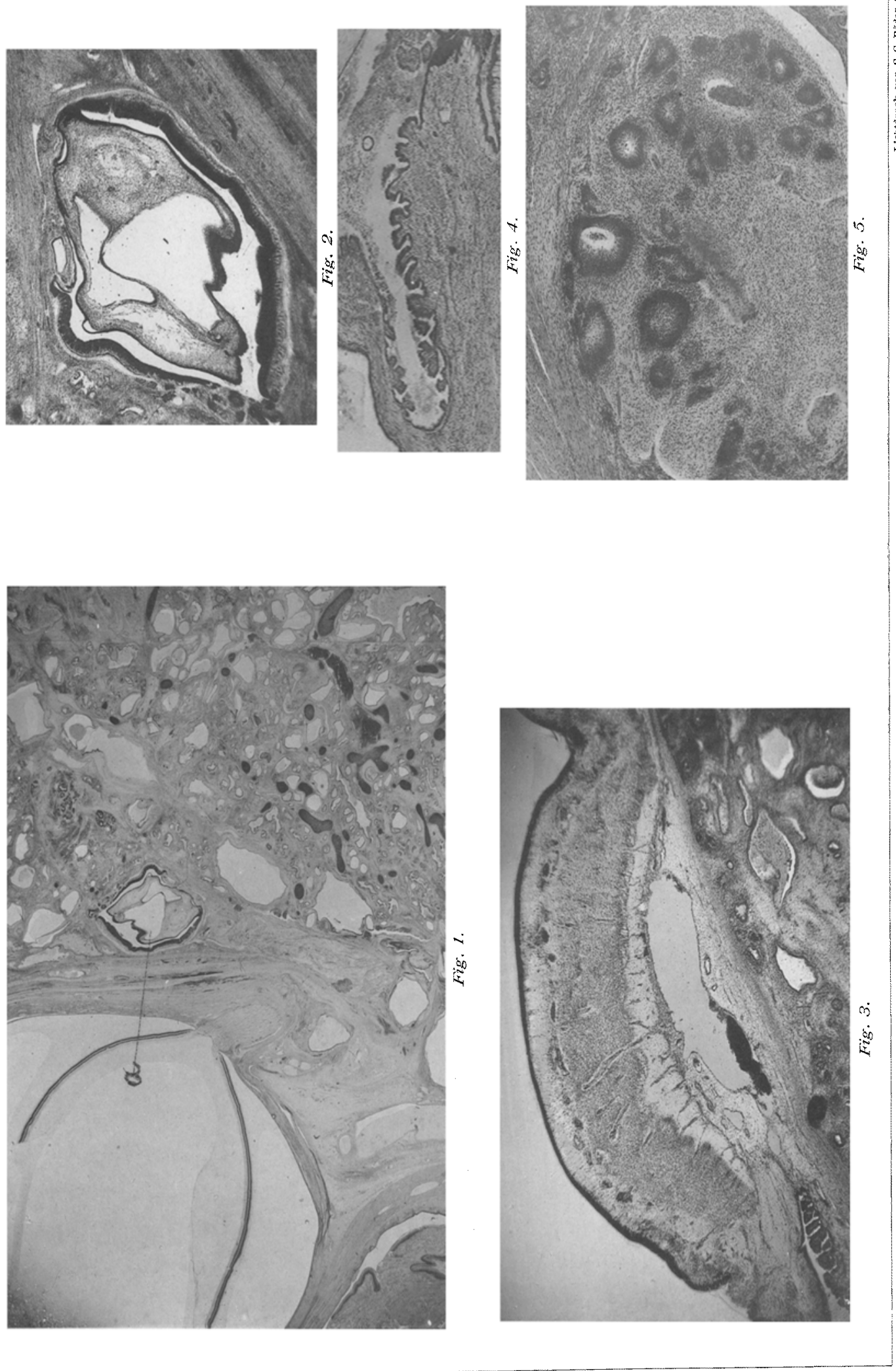

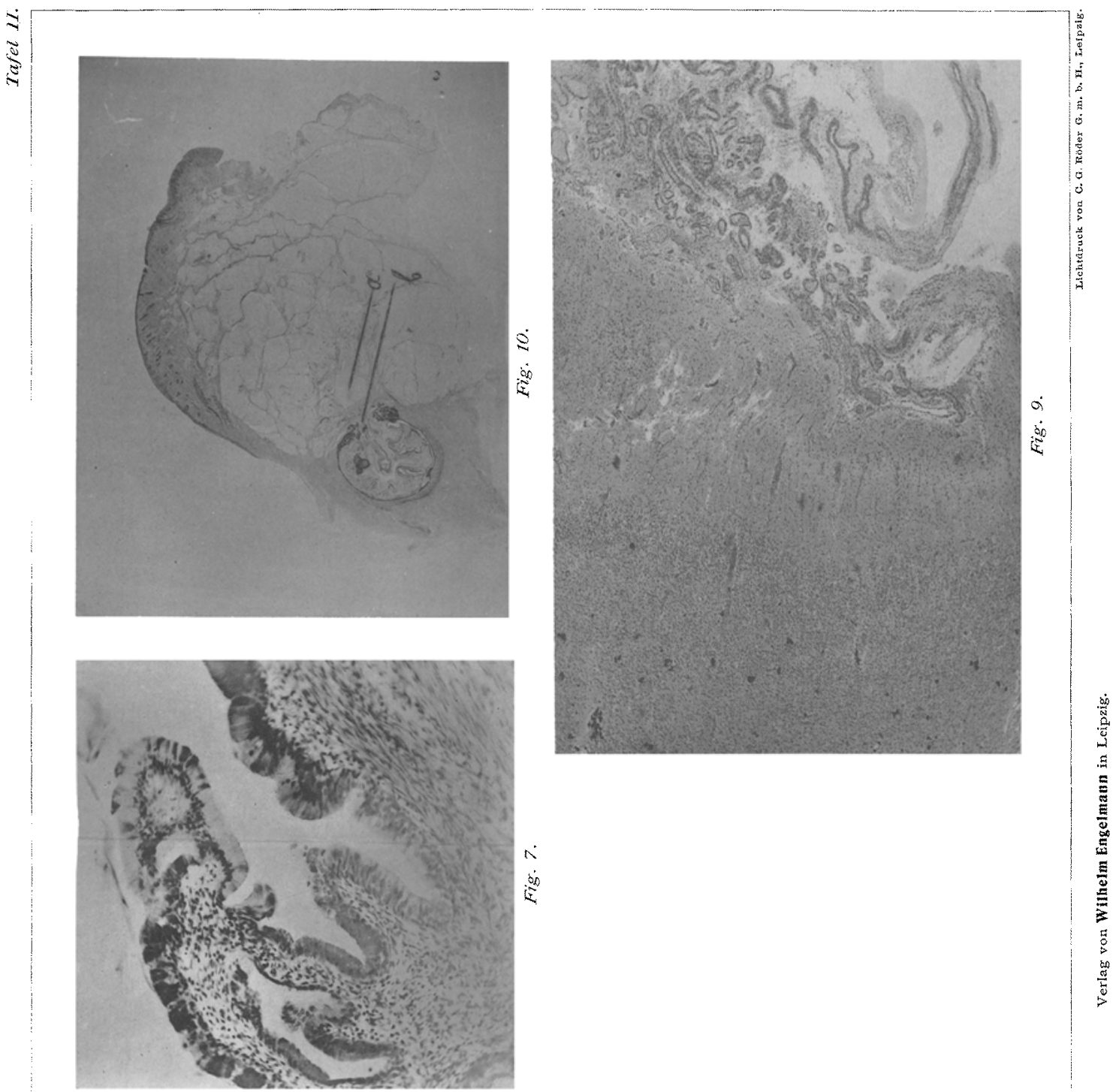

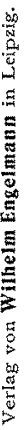
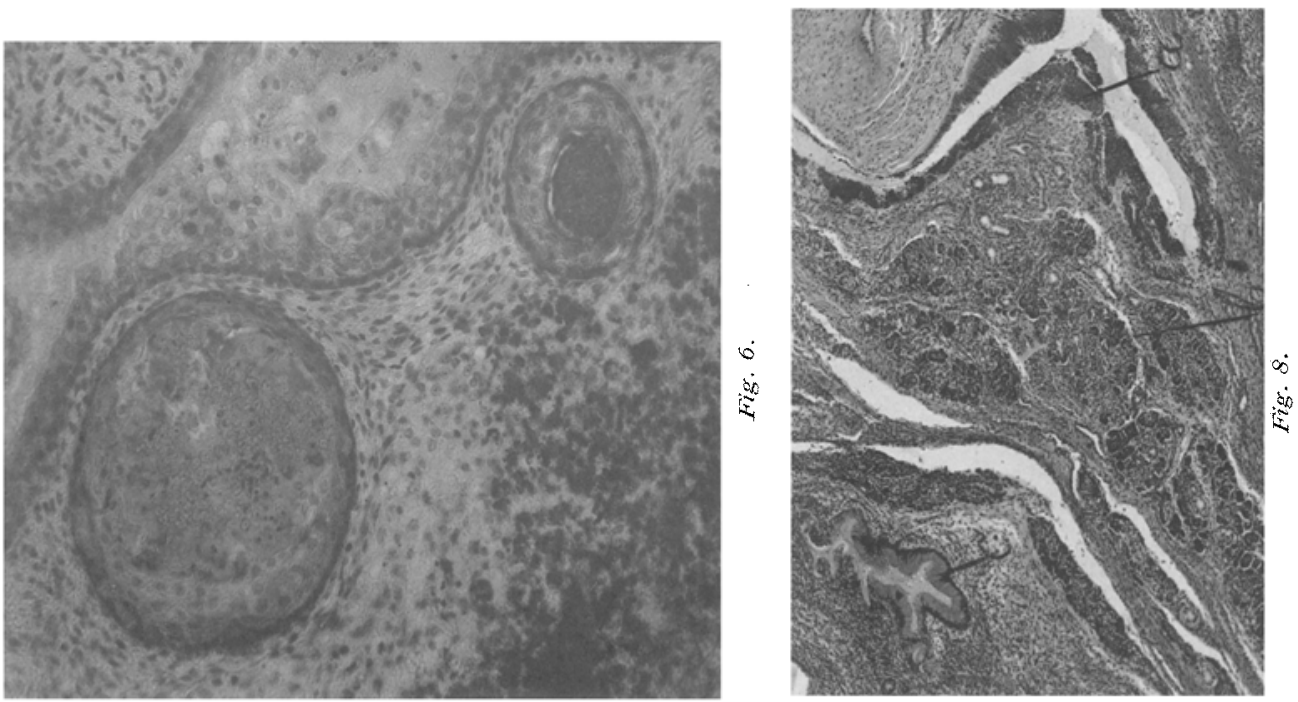


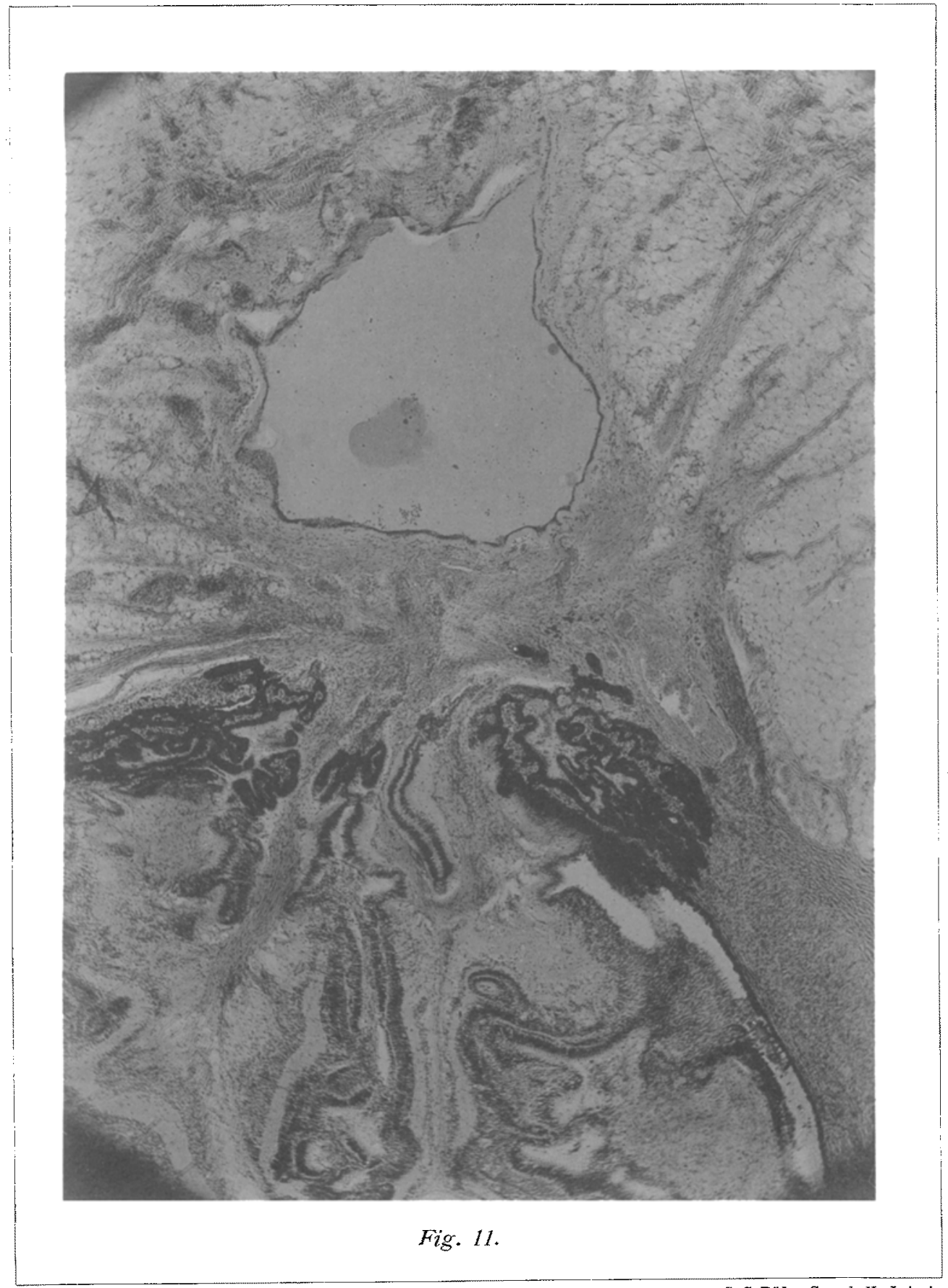

Lichtdrack ron C. G. Röder G. m. b. H., Leipzic. 